\title{
Decentralized Relay Selection in Multi-User Multihop Decode-and-Forward Relay Networks
}

\author{
Rajitha Senanayake, Member, IEEE, Saman Atapattu, Member, IEEE, \\ Jamie Evans, Senior Member, IEEE, and Peter Smith, Fellow, IEEE
}

\begin{abstract}
This paper analyzes the performance of a multiuser multihop relay network using a low complexity decentralized relay selection (DRS) scheme for decode-and-forward cooperative networks. We carry out a rigorous diversity order analysis, with Nakagami- $m$ fading and pathloss and show that the DRS scheme achieves full diversity while maintaining a complexity that is quadratic in the number of users, quadratic in the number of relays and independent of the number of hops. For a special case of two-user networks we derive exact closed-form expressions for the outage probability by considering the order statistics. Furthermore, we extend our analysis to consider interfering relay networks and derive an accurate lower bound on the outage of an arbitrary network user. Based on the lower bound we also show how the outage probability saturates in the high signal-tointerference-plus-noise ratio (SINR) regime. Extensive numerical examples are used to illustrate the accuracy of the analysis and to highlight the use of the DRS scheme in multi-user multihop relay networks.
\end{abstract}

Index Terms-Decode-and-forward, multihop networks, outage probability, relay selection.

\section{INTRODUCTION}

Cooperative communication networks - comprised of multiple nodes distributed across the network forming virtual multiple antenna arrays - have recently emerged as a promising technique to broaden network coverage, enhance network reliability, and mitigate the effect of fading in wireless communication networks [1]-[7]. Cooperative communication networks with multiple relay nodes, known as cooperative relay networks, typically arise in sensor mesh and ad hoc communications. For such networks, the careful selection of relays is extremely important in order to achieve full spatial diversity with low complexity and overhead. As a result, relay selection (RS) in cooperative relay networks has gained considerable research attention during the past few years.

Copyright (c) 2013 IEEE. Personal use of this material is permitted. However, permission to use this material for any other purposes must be obtained from the IEEE by sending a request to pubs-permissions@ieee.org.

Manuscript received August 16, 2017; revised December 02, 2017 and February 18, 2018; accepted February 20, 2018. The review of this paper was coordinated by Prof. Juan Romero-Jerez.

R. Senanayake, S. Atapattu and J. Evans are with the Department of Electrical and Electronic Engineering, University of Melbourne, Parkville, VIC. 3010, Australia (e-mail: rajitha.senanayake@unimelb.edu.au, saman.atapattu@unimelb.edu.au, jse@unimelb.edu.au).

P. Smith is with the School of Mathematics and Statistics, Victoria University of Wellington, Wellington, New Zealand (e-mail: peter.smith@ecs.vuw.ac.nz).

This work is supported in part by the Australian Research Council (ARC) through the Discovery Early Career Researcher (DECRA) Award DE180100501, DE160100020 and the Discovery Project DP140101050.

Digital Object Identifier xx.xxxx/TWC.2018.xxxx
There exists a rich body of literature on RS for two-hop networks where each source experiences a link from the source to relay and relay to destination [8]-[17]. There are various protocols proposed to choose the serving relay from among a collection of available relays, such as best relay selection where the relay whose path has the maximum signal-to-noise ratio (SNR) is selected [8], [16], nearest neighbor selection where the relay that is nearest to the base station is selected [10], best harmonic mean selection where the relay with the largest harmonic mean is selected [9]. In [17], authors consider a two-path relay channel where two relays take turns to decode-and-forward (DF) the transmissions from source to destination. All these works consider networks with single source-destination pairs. In [18], the authors extended RS to multi-user networks where, due to the user competition for relays, the RS problem becomes more challenging when compared to the single source-destination case. An optimal RS (ORS) algorithm which maximizes the minimum end-toend received SNR of all users is investigated. The complexity of ORS is quadratic in both the number of relays and the number of users. The authors also propose a suboptimal RS (SRS) scheme with complexity linear in the number of relays and quadratic in the number of users. Based on amplify-andforward (AF) relaying, the authors derive the diversity order of both the ORS and SRS schemes and compare it against the single source-destination case.

Recently, there has been increasing interest in multihop cooperative relay networks where the signal from source to the destination traverses through multiple clusters of relay nodes [19]-[25]. It is a promising technique to increase the transmission range in wireless networks. In [19], a novel optimization metric is derived to select the best relay on a per-hop basis. A suboptimal hop-by-hop RS is proposed that utilizes the mean channel state information (CSI) of the current hop. In [20], the authors present three RS schemes, optimal, adhoc and $\mathrm{N}$-hop, to achieve the full diversity gain provided by the cooperation between relays in each hop. Similar to the dual-hop network, in ORS, serving relays were selected to maximise the received SNR at the destination while in adhoc and N-hop schemes, RS is conducted independently in each hop and set of $N$ hops, respectively. Based on DF relaying, the outage analyses of the proposed three RS schemes were conducted for Rayleigh fading channels. In [21], an approximate lower bound on the average bit error rate (BER) for ORS was derived based on the probability density function (PDF) of a max-min exponential random variable. 
More recently in [22], the exact BER of ORS in Rayleigh fading was analyzed based on a convergent power series of gamma functions and a tight approximation of the Gaussian $Q$-function. It is again noted that the ORS is a centralized algorithm that selects the best relay path with a full diversity performance at the cost of high complexity and high signaling overheads to obtain CSI of all links. In [23]-[25], authors consider the interesting application of multihop relaying in cognitive radio networks and analyse the end-to-end outage probability. However, all aforementioned works over multihop relaying consider only a single source-destination pair.

When we have multiple source-destination pairs the amount of research work available in the open literature is limited. For such networks, spectral-efficient half-duplex transmission schemes with interference alignment were proposed in [26] to minimize the impact of the interference between the relays. However, the authors did not provide a RS scheme to select the multihop relay paths for each user pair. In [27], a lowcomplexity decentralized RS scheme is proposed for a twouser multihop relay network. Based on DF relaying, the authors derive exact closed-form expressions for the outage probability of each user and show that the proposed decentralized RS scheme can achieve full diversity. However, the results and the analysis is limited to the case with only two source-destination pairs. Thus, there remains a critical need to design new multihop RS algorithms for a more general multiuser network to select the best relays in each hop and assign these relays to each user pair. Finding such optimal paths is very challenging and our motivation is to design a multihop multiple RS strategy that can support multiple sourcedestination users with low overheads while still achieving a full diversity order.

In this paper, we consider a multi-user multihop relay network where each hop is equipped with multiple relays that assist users to communicate with their designated destinations. We assume that at each hop, each user can only be helped by a single relay and one relay can help at most one user. The main contributions of this paper are listed as follows.

- We propose a decentralized RS (DRS) algorithm for multi-user multihop relay networks, that maximizes the minimum received SNR or signal-to-interference-plusnoise ratio (SINR) for all users. In the ORS algorithm where the path through the relay network for each user is found by an exhaustive search, the end-to-end network performance is maximized. However, it requires global CSI and a joint optimization of all possible paths through the network, which adds significant processing complexity. Our proposed DRS is a more practical hop-by-hop routing scheme where RS is performed independently in each hop and the complexity is quadratic with the number of relays but independent of the number of hops.

- The diversity order of the proposed DRS algorithm is analyzed theoretically based on the DF relaying protocol. Considering a general Nakagami- $m$ fading model, it is shown that the DRS algorithm can achieve full spatial diversity, i.e., for a network with $N_{s}$ source nodes and $N$ relays, all source nodes have diversity order $m N$,

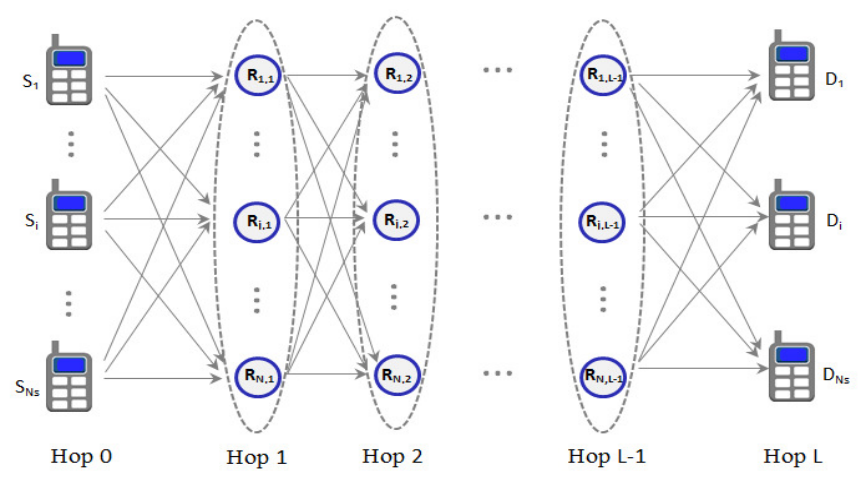

Fig. 1. A multi-user multihop relay network.

when receiving nodes have complete interference cancellation capability. Thus, with the DRS algorithm user competition for relays does not affect diversity order. Furthermore, we derive a closed-form expression for the array gain of the system. For the special case of two-user networks, an exact closed-form expression for the outage probability is derived using order statistics.

- We extend our analysis of the DRS algorithm to interfering multi-user multihop relay networks where the signals transmitted by active nodes in each hop interfere with each other. The introduction of interference causes the SINRs of users to be correlated with each other resulting in a more challenging mathematical problem. Based on the DRS algorithm we derive a tight lower bound on the outage probability of interfering users. We clearly illustrate how interference degrades the outage probability performance and using the lower bound we show that the outage probability enters a saturation regime at high SINRs.

- Extensive numerical examples are used to simulate the outage probability performance, illustrating the accuracy of our analytical derivations. Furthermore, the performance of our proposed DRS algorithm is compared against three performance benchmarks that were identified based on well-known RS algorithms, namely, optimal, naive and random RS [18]. Interestingly, we reveal that the performance gap between DRS and ORS is small for a low number of relays and hops.

The rest of the paper is organized as follows. The system model is presented in Section II. The RS schemes, including the ORS, naive RS and the proposed sub-optimal DRS, are introduced and detailed in Section III. In Section IV the analysis for DRS without user interference is provided along with the special case of two-user relay networks. The case with interfering relay networks is investigated in Section V. Finally, the numerical examples are provided in Section VI followed by the concluding remarks in Section VII.

\section{System Model}

We consider a multihop wireless relay network, shown in Fig. 1, where $N_{s}$ source nodes $\left(S_{1}, S_{2}, \ldots S_{N_{s}}\right)$ send information to $N_{s}$ corresponding destination nodes $\left(D_{1}, D_{2}, \ldots D_{N_{s}}\right)$ 
via a general $L$-hop network with $N$ DF relays in each hop. We have $N_{s}$ user pairs, denoted as user $u, u \in\left\{1,2, \ldots N_{s}\right\}$, where user $u$ corresponds to the $S_{u}-D_{u}$ pair. There are $(L+1)$ node clusters: a source cluster, $(L-1)$ relay clusters and a destination cluster. We assume that each user pair is assisted by only one relay in each cluster (and each relay assists at most one user) to minimize the synchronization requirement and avoid creating too much processing complexity in any single relay. Thus, we need $N \geq N_{s}$ relays in each cluster and the communication takes place between nodes in two neighboring clusters at a time. Each node is equipped with a single antenna and operates in half-duplex mode with a power budget of $P$ for each transmission. We denote the $i$ th node of the $l$ th hop as $R_{i, l}$ where $i=1,2, \ldots N_{s}$ for $l=0$ and $L$, and $i=1, \cdots, N$ for $l=1,2, \cdots, L-1$. Here, the 0th hop and $L$ th hop correspond to the source and destination clusters, respectively. In the first part of the paper, we consider an interference free network. As such, we assume either the active nodes in each hop are assigned orthogonal channels or receiving nodes have complete interference cancellation capabilities. In Section V, this assumption is removed allowing the users to interfere.

We consider DF relaying at the relays, and each relay has a sufficient SNR level for error-free decoding. Let $s_{i, l}$ denote the information symbol for transmission at $R_{i, l}$ which has unit average energy. The transmit signal undergoes both multipath fading and distance-dependent path loss ${ }^{1}$. The channel coefficient and distance between node $R_{i, l-1}$ and node $R_{j, l}$ are $h_{i j, l}$ and $d_{i j, l}$, respectively. The received signal at node $R_{j, l}$ from node $R_{i, l-1}$ can be written as

$$
y_{j, l}=\sqrt{\frac{P}{d_{i j, l}^{\beta}}} h_{i j, l} s_{i,(l-1)}+n_{j, l}
$$

where $\beta$ is the path loss exponent, and $n_{j, l}$ is the additive noise at $R_{j, l}$, which is assumed to be independent and identically distributed (i.i.d.) complex Gaussian with zero mean and $\sigma^{2}$ variance. We assume that the distance between hops is much larger than the distance between the relay nodes in each hop. Therefore, the channel gains between transmit nodes in the $(l-1)$ th hop and the receiving nodes in the $l$ th hop are i.i.d. However, the channel gains between different consecutive hops are independently but non-identically distributed., i.e., the channel gains between transmit nodes in the $(l-1)$ th hop and the receiving nodes in the $l$ th hop and the channel gains between transmit nodes in the $(k-1)$ th hop and the receiving nodes in the $k$ th hop, where $l \neq k$, are non-identically distributed.

The corresponding received SNR is given as

$$
\tilde{\gamma}_{i j, l}=\frac{P g_{i j, l}}{d_{i j, l}^{\beta} \sigma^{2}}
$$

where $g_{i j, l}=\left|h_{i j, l}\right|^{2}$. This SNR is valid for any hop $l=$ $1, \cdots, L$, and the SNR matrix of the each hop which includes

\footnotetext{
${ }^{1}$ We model large scale fading as a pure distance based path loss model. The absence of shadow fading is motivated by the fact that in a multihop relay network, relay nodes can be placed in such a way that shadowing is avoided.
}

all possible connections can be given as

$$
\tilde{\Gamma}_{l}= \begin{cases}\left(\tilde{\gamma}_{i j, l}\right) \in \mathbb{R}^{N_{s} \times N} ; \quad l=1 \\ \left(\tilde{\gamma}_{i j, l}\right) \in \mathbb{R}^{N \times N} ; \quad l=2, \cdots, L-1 \\ \left(\tilde{\gamma}_{i j, l}\right) \in \mathbb{R}^{N \times N_{s}} ; \quad l=L .\end{cases}
$$

We assume that the amplitude of the channel gain, $\left|h_{i j, l}\right|$, follows a Nakagami- $m$ distribution and all wireless channels are i.i.d. Then, $g_{i j, l}$ follows a gamma distribution, and the corresponding cumulative distribution function (CDF) of $\tilde{\gamma}_{i j, l}$ can be given as

$$
F_{\tilde{\gamma}_{i j, l}}(x)=\frac{\gamma\left(m, \frac{m x}{\tilde{\Omega}_{l}}\right)}{\Gamma(m)}
$$

where $m$ is a parameter related to the Nakagami fading channel, $\tilde{\Omega}_{l}=P /\left(d_{i j, l}^{\beta} \sigma^{2}\right)$, and $\Gamma($.$) and \gamma(.,$.$) are the gamma$ and incomplete gamma functions, respectively [28].

\section{Multi-hop Relay Selection Schemes}

The main challenge in a multi-user multihop relay network is to select the best path for each source to traverse through the network to the corresponding destination. In this section, we discuss possible RS schemes for a multi-user multihop network with multiple DF relays by focusing on the outage probability performance. It is important to note that for DF relaying the outage probability of a given path is limited by the minimum SNR of $L$ hops [20, eq. (3)]. Therefore, to minimize the outage probability of each user, the RS scheme should select the paths by maximising the minimum SNR of each user. Also, the RS scheme used for selecting relay nodes in each hop should be designed in such a way that user fairness is maintained while keeping the network complexity low. Considering the energy constraints at the relay node we make the practical assumption that relay nodes cannot be shared between users, i.e., a given relay node cannot serve more than one source node. In the following, we define RS schemes that take into account the individual performance as well as user fairness.

\section{A. Optimal RS Scheme and Challenges}

The ORS involves an exhaustive search through all possible paths from source nodes to destination nodes. If we consider user 1 who needs to pass through $L$ hops to reach its destination, we can find $N^{L-1}$ possible paths to traverse. Thus, for $N_{s}$ users we have $N_{s} N^{L-1}$ possible paths from source nodes to destination nodes. Since a relay cannot be shared between both users, for a given path assigned to one user, there are $(N-1)^{L-1}$ possible paths for the other user. Thus, we have $\left[\prod_{i=0}^{N_{s}-1}(N-i)\right]^{L-1}$ paths for $N_{s}$ users, and we need to find the $N_{s}$ paths that maximize the minimum end-to-end SNR of all users. Although this exhaustive search approach leads to an ORS scheme, it has an exponential complexity with the number of hops. For example, a small network with $N_{s}=2, N=3$ and $L=4$, results in 216 possible path pairs. Furthermore, the ORS scheme should be implemented by a centralized system with a master node (which may be an 
external node) that has access to global CSI of all the links in the network. This increases the complexity and overhead for channel training/estimation making the implementation of the ORS scheme virtually impossible.

\section{B. Naive RS Scheme}

In the naive RS, user 1 first selects the best path from among all possible paths. Then, user 2 selects its best path that does not conflict with user 1 and so on. In each hop, user $i$ selects one relay from among $(N-i)$ relays that are not used by the first $(i-1)$ users, i.e., user 1 to user $(i-1)$. As such, the user fairness is compromised, and in a system with Nakagami- $m$ fading channels user $i$ can only achieve a maximum diversity order of $(N-i) \times m$. However, the complexity of the selection algorithm is lower than the ORS scheme as we only have $N^{L-1}$ paths for user 1 and $(N-1)^{L-1}$ paths for user 2 and so on.

\section{Proposed Decentralized RS (DRS) Scheme}

In this paper, we consider a DRS scheme which performs RS independently at each hop. As opposed to the optimal and naive RS schemes, this scheme achieves user fairness while maintaining lower network complexity.

Note that, the $(l-1)$ th hop has a set of $N$ relays which we denote as $\mathcal{R}_{l-1}=\left\{R_{1, l-1}, \cdots, R_{N, l-1}\right\}$. After the $\mathrm{RS}$ at the $(l-1)$ th hop, a set of $N_{s}$ relays is selected from $\mathcal{R}_{l-1}$ and we denote this as $\mathcal{R}_{l-1}^{*}=\left\{R_{1, l-1}^{(1)}, \cdots, R_{u, l-1}^{(u)}, \cdots, R_{N_{s}, l-1}^{\left(N_{s}\right)}\right\}$ where $R_{u, l-1}^{(u)}$ is the selected relay for the user $u$ at the $(l-1)$ th hop. Then, the SNR matrix from $\mathcal{R}_{l-1}^{*}$ to $\mathcal{R}_{l}$ can be formed by first extracting the corresponding rows from $\tilde{\Gamma}_{l}$ in eq. (3), and then arranging them according to the user index $u$. Thus, the RS matrix at the $l$ th hop may be given as

$$
\boldsymbol{\Gamma}_{l}=\left(\gamma_{u j, l}\right) \in \mathbb{R}^{N_{s} \times N} ; l=1, \cdots, L
$$

It is important to note that $\boldsymbol{\Gamma}_{1}=\tilde{\boldsymbol{\Gamma}}_{1}$; for $l=2, \cdots, L-1$, the entry $\gamma_{u j, l}$ represents the SNR at $R_{j, l}$ given that $R_{u, l-1}^{(u)}$ is selected for the user $u$ at the $(l-1)$ th hop which can be extracted from entries in $\tilde{\boldsymbol{\Gamma}}_{l}$; and $\boldsymbol{\Gamma}_{L}=\tilde{\boldsymbol{\Gamma}}_{L}^{T}$ which is the transpose of $\tilde{\boldsymbol{\Gamma}}_{L}$. Based on $\boldsymbol{\Gamma}_{l}$, we perform the following steps.

- $R S$ at the lth hop $(l=1, \cdots, L-2)$ : We need to select $N_{s}$ relays at the $l$ th hop that will maximize the minimum SNR of $N_{s}$ users at the $l$ th hop. To do so, we apply the optimal single-hop RS algorithm proposed in [15] and extended in [18], in which the worst-case complexity is $\mathcal{O}\left(N_{s}^{2} N^{2}\right)$. While this $\mathrm{RS}$ algorithm is detailed for a dual-hop $(L=2)$ relay network in [15], for the sake of completeness, we include the main steps applicable for a general multi-hop ( $L \geq 2$ ) relay network, as given below.
Steps of the DRS scheme for $l=1, \cdots, L-2$

1. Generate $\Gamma_{l}$ using (5).

2. Sort all $\gamma_{u j, l}$ 's in descending order as $\gamma_{(1), l} \geq$ $\gamma_{(2), l} \geq \ldots \geq \gamma_{\left(N_{s} N\right), l}$, where $\gamma_{(k), l}$ denotes the $k$ th largest element among $N_{s} N$ independent entries in $\Gamma$.

3. Generate a temporary matrix $\hat{\boldsymbol{\Gamma}}_{l}$ with $N_{s}$ rows and $N$ columns. Fill $\hat{\boldsymbol{\Gamma}}_{l}$ starting from $\gamma_{(1), l}$ until each row and at least $N_{s}$ columns have entries. Having an entry in each row make sure that there is a selection available for each user. Having entries in at least $N_{s}$ columns make sure that the relays are not shared between users. This filling process results in maximizing the minimum SNR assigned to each user. 4. Assign the last entry for the corresponding user and remove the row and the column.

5. Repeat step 4 until all users have a relay assigned. If an assignment cannot be made go back to step 3 and add the next entry to $\hat{\Gamma}_{l}$.

6. Repeat steps 4 and 5 until all users have assigned relays.

We apply this selection criterion for each SNR matrix sequentially starting from $\boldsymbol{\Gamma}_{1}$ to $\boldsymbol{\Gamma}_{L-2}$. As such, the complexity of each selection is quadratic in the number of relays but independent of the number of hops.

- RS at the $(L-1)$ th hop: In the final two clusters of relays (i.e., the $(L-1)$ th and $L$ th hop), we perform a joint RS by using $\boldsymbol{\Gamma}_{L-1}$ in (5) and $\tilde{\boldsymbol{\Gamma}}_{L}$ in (3) and construct $\boldsymbol{\Gamma}_{L-1, L}$ as

$$
\boldsymbol{\Gamma}_{L-1, L}=\min \left(\gamma_{u j, L-1}, \tilde{\gamma}_{j u, L}\right) \in \mathbb{R}^{N_{s} \times N} .
$$

We repeat the algorithm for the $(L-1)$ th hop by using $\boldsymbol{\Gamma}_{L-1, L}$. The motivation for the joint RS is because if we apply the RS algorithm directly to $\boldsymbol{\Gamma}_{L-1}$, we will select $N_{s}$ relays in the $(L-1)$ th hop, and the selected relays will directly communicate with the corresponding destination nodes in the $L$ th hop. As such, $\tilde{\boldsymbol{\Gamma}}_{L}$ is not involved in the selection which results in a degradation in the diversity order [20].

Our proposed DRS scheme can be implemented in a decentralized manner where a master node is assigned in each hop (which may be one of the relays) that has access to local CSI of the links in the given hop. Thus, the DRS scheme will lead to a significant decrease in the complexity and overhead compared with the ORS scheme based on exhaustive search.

\section{Examples}

We consider a network with $N_{s}=2, N=2$ and $L=3$. Each relay cluster has two relays $N=2$. For one channel realization, we assume the SNR matrices for three hops $\tilde{\boldsymbol{\Gamma}}_{1}$, $\tilde{\boldsymbol{\Gamma}}_{2}$ and $\tilde{\boldsymbol{\Gamma}}_{3}$, respectively, are given by
$\left[\begin{array}{l}0.52 \\ 0.40\end{array}\right.$
$\left.\begin{array}{l}0.69 \\ 0.78\end{array}\right] ; \quad\left[\begin{array}{l}0.58 \\ 0.07\end{array}\right.$
$\left.\begin{array}{l}0.97 \\ 0.45\end{array}\right] ;$ and $\left[\begin{array}{ll}0.03 & 0.62 \\ 0.10 & 0.44\end{array}\right]$

- Optimal RS: Applying an exhaustive search, we have selections $S_{1}-R_{2,1}-R_{2,2}-D_{1}$ and $S_{2}-R_{1,1}-R_{1,2}-D_{2}$. 
Then, the effective end-to-end SNRs are 0.10 for user 1 and 0.40 for user 2, where the minimum SNR of the network is 0.10 .

- Naive RS: Applying the naive RS scheme, we have selections $S_{1}-R_{1,1}-R_{2,2}-D_{1}$ and $S_{2}-R_{2,1}-R_{1,2}-D_{2}$. Then, the effective end-to-end SNRs are 0.10 for user 1 and 0.07 for user 2, where the minimum SNR of the network is 0.07 .

- Proposed DRS: To maximize the minimum SNR of $\boldsymbol{\Gamma}_{1}$, we select $R_{1,1}$ for $S_{1}$ and $R_{2,1}$ for $\mathrm{S} 2$. For the last two hops, we maximize the minimum SNR by using the joint selection based on $\boldsymbol{\Gamma}_{2}$ and $\tilde{\boldsymbol{\Gamma}}_{3}$. Then, we have selections $S_{1}-R_{1,1}-R_{2,2}-D_{1}$ and $S_{2}-R_{2,1}-R_{1,2}-D_{2}$. The effective end-to-end SNRs are 0.10 for user 1 and 0.07 for user 2, where the minimum SNR is 0.07.

- Proposed DRS without joint selection: To maximize the minimum SNR of $\boldsymbol{\Gamma}_{1}$ and $\boldsymbol{\Gamma}_{2}$, we select $R_{1,1}$ for $S_{1}$ and $R_{2,1}$ for $S_{2}$; and $R_{1,2}$ for $R_{1,1}$ and $R_{2,2}$ for $R_{2,1}$ which means, so far, we have selections $S_{1}-R_{1,1}-R_{1,2}$ and $S_{2}-R_{2,1}-R_{2,2}$. Since there are no remaining options in the last hop, $R_{1,2}$ transmits to $D_{1}$ and $R_{2,2}$ transmits to $D_{2}$. As such, the effective end-to-end SNRs are 0.03 for user 1 and 0.44 for user 2, where the minimum SNR is 0.03. This highlights the performance advantage of joint selection in the last two hops.

Interestingly, if we consider the channel realizations of the SNR matrices $\tilde{\boldsymbol{\Gamma}}_{1}, \tilde{\boldsymbol{\Gamma}}_{2}$ and $\tilde{\boldsymbol{\Gamma}}_{3}$, respectively, are given by

$$
\left[\begin{array}{ll}
0.60 & 0.89 \\
0.82 & 0.23
\end{array}\right] ;\left[\begin{array}{ll}
0.16 & 0.52 \\
0.69 & 0.49
\end{array}\right] ; \text { and }\left[\begin{array}{cc}
0.86 & 0.85 \\
0.11 & 0.79
\end{array}\right]
$$

a minimum SNR of the network of 0.52 is achieved for all three RS schemes. This illustrates that for some example scenarios both naive RS and proposed DRS give optimal results. But for many other example scenarios the minimum SNR of the network with naive RS or proposed DRS is smaller that that of the ORS. Hence, naive RS and proposed DRS are sup-optimal schemes.

\section{Performance Analysis For DRS without USER-INTERFERENCE}

In this section, we first derive the outage probability, diversity order and array gain of a general multihop network based on the DRS scheme discussed in Section III. Then, we consider the performance of a two-user network as a special case.

\section{A. Outage Probability}

For notational simplicity, let us define the effective SNR between nodes $R_{i, l-1}$ and $R_{j, l}$ as $\dot{\gamma}_{i j, l}$. Note that, for $l=$ $1, \cdots, L-2$, we can write that $\dot{\gamma}_{i j, l}=\tilde{\gamma}_{i j, l}$ where $\tilde{\gamma}_{i j, l}$ is in (2). In the last two hops, i.e., hop $L-1$ and $L$, we apply the joint selection such that the SNR of the channel between nodes $R_{i, L-2}$ and $R_{j, L}$ via $R_{k, L-1}$ can be given by $\min \left(\tilde{\gamma}_{i k, L-1}, \tilde{\gamma}_{k j, L}\right)$. Recall that $j \in\left\{1,2, \ldots, N_{s}\right\}$ is for the $N_{s}$ destination nodes. Then, we denote the effective SNR of the $(L-1)$ th hop as $\dot{\gamma}_{i j, L-1}=\min \left(\tilde{\gamma}_{i k, L-1}, \tilde{\gamma}_{k j, L}\right)$. Based on (4), we can derive the corresponding CDF of $\hat{\gamma}_{i j, l}$ as

$$
\begin{aligned}
& F_{\dot{\gamma}_{i j, l}}(x) \\
& =\left\{\begin{array}{lr}
F_{\tilde{\gamma}_{i j, l}}(x) ; & l=1, \cdots, L-2 \\
1-\left(1-F_{\tilde{\gamma}_{i j, L-1}}(x)\right)\left(1-F_{\tilde{\gamma}_{i j, L}}(x)\right) ; & l=L-1 .
\end{array}\right.
\end{aligned}
$$

Here the expression for $l=L-1$ results due to the joint processing in the last two hops. For presentation simplicity, we assume that the $(L-1)$ relay clusters are placed between the source and destination pairs with equal distances, i.e., $d_{i j, l}=$ $d / L ; \forall i, j, l$ where $d$ is the distance between the source and destination pairs. Since all channels are identical, we set $\tilde{\Omega}_{l}=$ $\Omega=P /\left((d / L)^{\beta} \sigma^{2}\right)$ for $l=1, \cdots, L$. However, it is important to note that the following derivation applies to the general case where the $(L-1)$ relay clusters are placed randomly between the source and destination pairs such that the distance between clusters in not identical.

Since we maximize the minimum SNR for each hop in the channel matrix we need to apply the order statistics of $\dot{\gamma}_{i j, l}$. The CDF of the $k$ th largest element among $N_{s} N$ independent entries in $\boldsymbol{\Gamma}_{l}$, denoted as $\gamma_{(k), l}$, can be written as [18, eq. 8]

$$
F_{\gamma_{(k), l}}(x)=\sum_{i=0}^{k-1} \frac{\left(N_{s} N\right) !\left(\begin{array}{c}
k-1 \\
i
\end{array}\right)(-1)^{i} F_{\dot{\gamma}_{i j, l}}(x)^{N_{s} N-k+i+1}}{\left(N_{s} N-k+i+1\right)\left(N_{s} N-k\right) !(k-1) !}
$$

where $F_{\dot{\gamma}_{i j, l}}(x)$ is chosen from (6) depending on the hop. For the DF multihop relay network the end-to-end SNR of user $u$, $\gamma^{(u)}$, can be written as

$$
\gamma^{(u)}=\min \left\{\gamma_{u, 1}, \cdots, \gamma_{u, l}, \cdots, \gamma_{u, L-1}\right\},
$$

where $\gamma_{u, l}$ is the selected SNR for user $u$ at the $l$ th hop, i.e, the SNR selected from among $N_{s}$ SNRs in the $u$ th row of $\boldsymbol{\Gamma}_{l}$. With the DRS scheme, we can write an expression for the outage probability of user $u$ such that the end-to-end SNR falls below a certain predetermined threshold SNR $\gamma_{t h}$ as

$$
\begin{aligned}
P_{o}^{(u)} & =\mathbb{P}\left[\gamma^{(u)} \leq \gamma_{t h}\right] \\
& =\mathbb{P}\left[\min \left\{\gamma_{u, 1}, \cdots, \gamma_{u, l}, \cdots, \gamma_{u, L-1}\right\} \leq \gamma_{t h}\right] \\
& =1-\prod_{l=1}^{L-1}\left(1-\mathbb{P}\left[\gamma_{u, l} \leq \gamma_{t h}\right]\right) .
\end{aligned}
$$

Based on our DRS scheme which maximizes the minimum SNR for each $\boldsymbol{\Gamma}_{l}$, we note that $\gamma_{u, l}$ can take $\gamma_{(1), l}, \gamma_{(2), l}, \cdots$, or $\gamma_{\left(N_{s} N-N+1\right), l}$. Thus, we can re-write (9) as

$$
\begin{aligned}
& P_{o}^{(u)} \\
& =1-\prod_{l=1}^{L-1}\left(1-\sum_{k=1}^{N_{s}} \sum_{l=1}^{N-N+1} \mathbb{P}\left[\gamma_{u, l}=\gamma_{(k), l}\right] \mathbb{P}\left[\gamma_{(k), l} \leq \gamma_{t h}\right]\right) \\
& =1-\prod_{k=1}^{L-1}\left(1-\sum_{s=1}^{N-N+1} \mathbb{P}\left[\gamma_{u, l}=\gamma_{(k), l}\right] F_{\gamma_{(k), l}}\left(\gamma_{t h}\right)\right)
\end{aligned}
$$

where $F_{\gamma_{(k), l}}\left(\gamma_{t h}\right)$ is defined in (7) and $\mathbb{P}\left[\gamma_{u, l}=\gamma_{(k), l}\right]$ denotes the probability of each event $\gamma_{u, l}=\gamma_{(k), l}$ where 
$k=1, \cdots, N_{s} N-N+1$. For the general case with $N_{s}$ users, deriving analytical expressions for the probability of each event is challenging, however, these probabilities can be calculated numerically, via Monte Carlo simulations. We provide analytical expressions of $\mathbb{P}\left[\gamma_{u, l}=\gamma_{(k), l}\right]$ s only for a two-user network in Section IV-C.

\section{B. Diversity Order and Array Gain}

Diversity order determines how fast the outage probability decreases in the high transmit power regime. It is defined as $\delta \triangleq \lim _{P \rightarrow \infty} \frac{\log P_{o}^{(u)}}{\log P}$ [29]. Then the array gain can be defined as $\epsilon=\lim _{P \rightarrow \infty}\left(P^{\delta} P_{o}^{(u)}\right)^{-1}$. In this section, we analyze the achievable diversity order and the array gain of the DRS scheme.

Theorem 1. In a multi-user multihop relay network with the DRS scheme, the achievable diversity order $\delta$ and array-gain $\epsilon$ of each user, respectively, are given by

$$
\begin{gathered}
\delta=m N \\
\epsilon=\left\{\begin{array}{cl}
\frac{\left(L-2+2^{N}\right)\left(N_{s} N\right) ! m^{m N} \prod_{i=1}^{N-1} \frac{N-i}{N_{s} N-i}}{N_{s} N !\left(N_{s} N-N\right) !(m !)^{N}\left(\gamma_{t h}\left(\frac{d}{L}\right)^{\beta} \sigma^{2}\right)^{-m N}} ; & N_{s}<N \\
\frac{2\left(L-2+2^{N}\right)\left(N_{s} N\right) ! m^{m N} \prod_{i=1}^{N-1} \frac{N-i}{N_{s} N-i}}{N_{s} N !\left(N_{s} N-N\right) !(m !)^{N}\left(\gamma_{t h}\left(\frac{d}{L}\right)^{\beta} \sigma^{2}\right)^{-m N}} ; & N_{s}=N,
\end{array}\right.
\end{gathered}
$$

where $m$ is the Nakagami shape parameter and $N$ is the number of relays in each hop.

A detailed proof of Theorem 1 is given in Appendix A. From Theorem 1 we understand that our DRS scheme achieves full diversity order.

If we do not perform joint processing in the last two hops, i.e., if the signal in the last hop is transmitted directly to the corresponding destination node without any node selection, then $\dot{\gamma}_{i j, l}=\tilde{\gamma}_{i j, l}$ for all the hops and we get $F_{\dot{\gamma}_{i j, l}}(x)=$ $F_{\tilde{\gamma}_{i j, l}}(x), \forall l \in\{1,2, \ldots, L\}$. Thus, the outage probability expression in (10) reduces to

$$
\begin{aligned}
P_{o}^{(u)} & =1-\left(1-\gamma\left(m, \frac{m \gamma_{t h}}{\tilde{\Omega}_{l}}\right)\right) \\
& \times \prod_{l=1}^{L-1}\left(1-\sum_{k=1}^{N_{s} N-N+1} \mathbb{P}\left[\gamma_{u, l}=\gamma_{(k), l}\right] F_{\gamma_{(k), l}}\left(\gamma_{t h}\right)\right) .
\end{aligned}
$$

Following the same steps as given in Appendix A, we can separate the higher order terms in (11) as

$$
P_{o}^{(u)}=\frac{\left(m \gamma_{t h}\left(\frac{d}{L}\right)^{\beta} \sigma^{2}\right)^{m}}{m !} P^{-m}+\mathcal{O}\left(\frac{1}{P^{m+1}}\right),
$$

and observe that without joint processing the diversity order reduces to $m$.

\section{Special Case: Two-User Networks}

In this section, we consider a special case with only two users and $N \geq 2$ relays. Then, the RS matrix of the network can be written as

$$
\boldsymbol{\Gamma}_{l}=\left[\begin{array}{ccccc}
\gamma_{11, l} & \cdots & \gamma_{1 n, l} & \cdots & \gamma_{1 N, l} \\
\gamma_{21, l} & \cdots & \gamma_{2 n, l} & \cdots & \gamma_{2 N, l}
\end{array}\right]_{2 \times N} .
$$

When $N_{s}=2$, the outage probability can be written by using (10) as

$$
P_{o}^{(u)}=1-\prod_{l=1}^{L-1}\left(1-\sum_{k=1}^{N+1} \mathbb{P}\left[\gamma_{u, l}=\gamma_{(k), l}\right] F_{\gamma_{(k), l}}\left(\gamma_{t h}\right)\right) .
$$

Now we consider the analytical expressions for $\mathbb{P}\left[\gamma_{u, l}=\right.$ $\left.\gamma_{(k), l}\right]$. Although they are presented in [27], for the sake of completeness, we briefly summarize the results as follows.

It is important to note that each event occurs with equal probability for each user pair $u$. Hence, we use a factor $1 / 2$ to calculate the outage of a particular user pair $u$. Using $\gamma_{\max }$ and $\gamma_{\min }$ to denote the maximum and minimum of the selected SNRs, we consider the probability of the following cases:

- $\gamma_{u, l}=\gamma_{(1), l}$ happens only if $\gamma_{\max }=\gamma_{(1), l}$, and thus $\gamma_{\min }$ can take any value of $\gamma_{(2), l}, \gamma_{(3), l}, \ldots$, or $\gamma_{(N+1), l}$. Subsequently, we consider the following three scenarios: i) $\gamma_{\max }=\gamma_{(1), l}$ and $\gamma_{\min }=\gamma_{(2), l}$ happens when $\gamma_{(1), l}$ and $\gamma_{(2), l}$ are in two distinct rows and two distinct columns of $\Gamma$. This event has probability $\frac{1}{2} \frac{N-1}{2 N-1}$; ii) $\gamma_{\max }=\gamma_{(1), l}$ and $\gamma_{\min }=\gamma_{(3), l}$ happens when $\gamma_{(1), l}$ and $\gamma_{(2), l}$ are in the same column, or $\gamma_{(1), l}$ and $\gamma_{(2), l}$ are in the same row and $\gamma_{(3), l}$ in the other row and a column different to $\gamma_{(1), l}$. This event has probability $\frac{1}{2} \frac{N}{2(2 N-1)}$;and iii) For $N>2, \gamma_{\max }=\gamma_{(1), l}$ and $\gamma_{\min }=\gamma_{(k), l}(k=4,5, \ldots, N+1)$ happens when all $\gamma_{(2), l}, \gamma_{(3), l}, \ldots, \gamma_{(k-1), l}$ are in the same row and $\gamma_{(k), l}$ in the other row and a column different to $\gamma_{(1), l}$. This event has probability $\frac{1}{2} \frac{N-1}{2 N-(k-1)} \prod_{i=1}^{k-2} \frac{N-i}{2 N-i}$.

- $\gamma_{u, l}=\gamma_{(2), l}$ happens when $\gamma_{\max }=\gamma_{(2), l}$ or $\gamma_{\min }=$ $\gamma_{(2), l}$. Since we have only two source nodes, $\gamma_{\max }$ can either be $\gamma_{\max }=\gamma_{(1), l}$ or $\gamma_{\max }=\gamma_{(2), l}$. Thus, $\mathbb{P}\left[\gamma_{\max }=\gamma_{(2), l}\right]=\frac{1}{2}-\mathbb{P}\left[\gamma_{u, l}=\gamma_{(1), l}\right]$ which can be calculated with the aid of the previous case. Further, $\gamma_{\min }=\gamma_{(2), l}$ happens only when $\gamma_{(1), l}$ and $\gamma_{(2), l}$ are in two distinct rows and two distinct columns of $\boldsymbol{\Gamma}_{l}$. This event has probability $\frac{1}{2} \frac{N-1}{2 N-1}$.

- $\gamma_{u, l}=\gamma_{(3), l}$ is equivalent to $\gamma_{\min }=\gamma_{(3), l}$ which happens when $\gamma_{(1), l}$ and $\gamma_{(2), l}$ are in the same column, or $\gamma_{(1), l}$ and $\gamma_{(2), l}$ are in the same row and $\gamma_{(3), l}$ is in a different row. This event has probability $\frac{1}{2} \frac{N+2}{2(2 N-1)}$.

- For $N>2, \gamma_{u, l}=\gamma_{(k), l}$ for $k=4, \cdots, N+1$ is equivalent to $\gamma_{\min }=\gamma_{(k), l}$ for $k=4, \cdots, N+1$ which happens when all $\gamma_{(1), l}, \gamma_{(2), l}, \cdots, \gamma_{(k-1), l}$ are in the same row and $\gamma_{(k), l}$ is in a different row. This event has probability $\frac{1}{2} \frac{2 N\left(\begin{array}{c}N \\ k-1\end{array}\right)}{(2 N-(k-1))\left(\begin{array}{c}2 N \\ k-1\end{array}\right)}$.

By considering all possible cases and substituting the corresponding event probabilities in (14), the exact outage probability of user $u, P_{o}^{(u)}$, can be derived in closed-form as (15), given at the top of next page. Moreover, following the similar steps as in Section IV-B, we can derive the diversity order and 


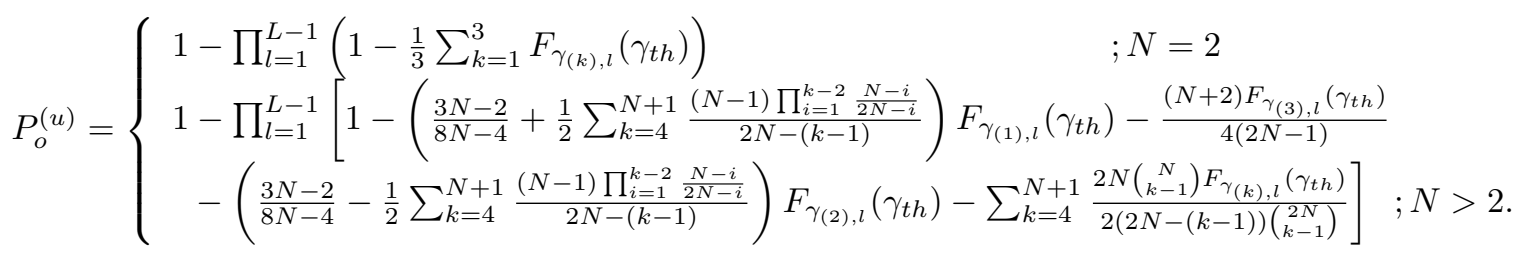

array-gain as

$$
\begin{gathered}
\delta=m N \\
\epsilon=\left\{\begin{array}{cl}
\frac{\left(L-2+2^{N}\right)(2 N) ! m^{m N} \prod_{i=1}^{N-1} \frac{N-i}{2 N-i}}{(N !)^{2}(m !)^{N}\left(\gamma_{t h}\left(\frac{d}{L}\right)^{\beta} \sigma^{2}\right)^{-m N}} ; & N=2 \\
\frac{\left(L-2+2^{N}\right)(2 N) ! m^{m N} \prod_{i=1}^{N-1} \frac{N-i}{2 N-i}}{2(N !)^{2}(m !)^{N}\left(\gamma_{t h}\left(\frac{d}{L}\right)^{\beta} \sigma^{2}\right)^{-m N}} ; & N>2,
\end{array}\right.
\end{gathered}
$$

This results is also consistent with Theorem 1 when $N_{s}=2$.

\section{Performance Analysis For DRS With USER-INTERFERENCE}

In this section, we consider a network with interfering users. Note that so far in this paper, we have assumed that the active nodes in each hop are assigned orthogonal channels or the receiving nodes have complete interference cancellation capability. As a result, the signal received by each user at each relay node was interference free. However, in a multiple-access system or a frequency-reused cell, interference from other transmitting sources may cause performance degradation and, therefore, cannot be ignored. When the users are interfering with each other the corresponding received signal at node $R_{j, l}$ can be written as

$$
y_{j, l}=\sum_{u=1}^{N_{s}} \sqrt{\frac{P}{\left(d_{u j, l}^{(u)}\right)^{\beta}}} h_{u j, l}^{(u)} s_{u,(l-1)}^{(u)}+n_{j, l},
$$

where $d_{u j, l}^{(u)}$ and $h_{u j, l}^{(u)}$ denotes the channel coefficients between node $R_{j, l}$ and $R_{u, l-1}^{(u)}$ and $s_{u, l-1}^{(u)}$ is the information symbol at $R_{u, l-1}^{(u)}$. The corresponding received SINR of user $u$ at node $j$ in hop $l$ is given as

$$
\gamma_{u j, l}=\frac{\omega_{u j, l}}{\sum_{q=1, q \neq u}^{N_{s}} \omega_{q j, l}+\sigma^{2}},
$$

where $\omega_{q j, l}=P g_{q j, l}^{(u)} /\left(d_{q j, l}^{(u)}\right)^{\beta}$ with $g_{q j, l}^{(u)}=\left|h_{q j, l}^{(u)}\right|^{2}$. From (17), we learn that the received SINRs of different users at a given node, i.e., the SINRs in each column of (5), are now correlated due to the effect of interfering channels.

Based on the proposed DRS algorithm, the outage probability of user $u$ can be found from (10), even in the interfering case. However, the analytical expression for $F_{\gamma_{(k), l}}\left(\gamma_{t h}\right)$ in (17) is no longer valid as $F_{\gamma_{(k), l}}\left(\gamma_{t h}\right)$ in the interfering case corresponds to the CDF of the $k$ th largest SINR from among $N_{s} N$ correlated SINRs. As discussed below, deriving an expression for $F_{\gamma_{(k), l}}\left(\gamma_{t h}\right)$ is mathematically challenging due to the complexity of resulting order statistics. Based on [30, eq. 5.3.1], the CDF of the $k$ th largest element among $N_{s} N$ correlated SINRs, denoted as $\gamma_{(k), l}$, can be written as

$$
F_{\gamma_{(k), l}}(x)=\sum_{j=N_{s} N-k+1}^{N_{s} N}(-1)^{j-N_{s} N+k-1}\left(\begin{array}{c}
j-1 \\
N_{s} N-k
\end{array}\right) H_{j: j}(x),
$$

where

$$
H_{j: j}(x)=\sum_{1 \leq i_{j+1}<\ldots<i_{N_{s} N} \leq N_{s} N} F_{\gamma_{(1), l}}^{i_{j+1, \ldots, i_{s} N}}(x),
$$

with $F_{\gamma_{(1), l}}^{i_{j+1, \ldots, N_{s} N}}(x)$ denoting the CDF of the largest of $j$ correlated random variables picked from the sample of $N_{s} N$ SINRs in the $l$ th hop. Let us denote this set of $j$ correlated random variables picked from the sample of $N_{s} N$ SINRs by $\gamma_{i_{1}, l}, \ldots, \gamma_{i_{j}, l}$. The superscript notation in (19) indicates that $\gamma_{i_{j+1}, l}, \ldots, \gamma_{i_{N_{s}}, l}$ have been dropped from the sample of $N_{s} N$ SINRs. By noting that $F_{\gamma_{(1), l}}^{i_{j+1, \ldots, N_{s} N}}(x)=$ $F_{\gamma_{i_{1}, l}, \ldots, \gamma_{i_{j}, l}}(x, \ldots, x)$, where $F_{\gamma_{i_{1}, l}, \ldots, \gamma_{i_{j}, l}}(x, \ldots, x)$ denotes the joint CDF of the selected $j$ SINRs, we can reexpress (18) as

$$
\begin{aligned}
F_{\gamma_{(k), l}}(x) & =\sum_{j=N_{s} N-k+1}^{N_{s} N}\left[(-1)^{j-N_{s} N+k-1}\left(\begin{array}{c}
j-1 \\
N_{s} N-k
\end{array}\right)\right. \\
& \left.\times \sum_{1 \leq i_{1}<\ldots<i_{j} \leq N_{s} N} F_{\gamma_{i_{1}, l}, \ldots, \gamma_{i_{j}, l}}(x, \ldots, x)\right] .
\end{aligned}
$$

As such, we need to know the joint CDF of all possible subsets of SINRs in $\Gamma_{l}$ in order to compute the CDFs of ordered SINRs. This is explained in detail in Appendix B using a simple example of $N_{s}=2$ and $N=2$. As a result deriving a general expression for $F_{\gamma_{(k), l}}(x)$, and therefore for outage probability, is mathematically challenging in the interfering case. As the exact results are intractable, in the following, we derive an accurate lower bound on the outage probability of an arbitrary user in interfering relay networks.

Remark: In the interfering case, all hops are processed individually, including the last two hops that were jointly analysed in the interference free case. This is because in the interfering case the SINRs in the $L$ th hop depends on the relay nodes that were selected in the $(L-1)$ th hop. As such, the master node has to consider all possible combinations of selected relay nodes in the $(L-1)$ th hop and compare the SINRs. This significantly increases the processing complexity. Furthermore, the effect of interference causes the performance to saturate in the high SINR regime. Therefore the loss of diversity gain that motivated the joint processing in the interference free case does not apply in the interfering case. 


\section{A. Lower Bound for Outage Probability}

From (17), we learn that each term in a given column in $\boldsymbol{\Gamma}_{l}$ is correlated and the elements in a given row are independent. Let $\gamma_{(N), l}^{(u)}$ denote the best SINR from among all the SINRs in the $u$ th row of $\boldsymbol{\Gamma}_{l}$, i.e., $\gamma_{(N), l}^{(u)}=\max \left(\gamma_{u 1, l}, \gamma_{u 2, l}, \ldots, \gamma_{u N, l}\right)$. If $\gamma_{(N), l}^{(u)}$ is assigned for user $u, \forall u \in\left\{1,2, \ldots, N_{s}\right\}$ then all the users experience the best SINR at hop $l$. However, this happens only if $\gamma_{(N), l}^{(1)}, \gamma_{(N), l}^{(2)}, \ldots, \gamma_{(N), l}^{\left(N_{s}\right)}$ are in different columns in $\Gamma_{l}$, because according to the proposed DRS scheme relays cannot be shared among users. Observing the structure of the SINR expression in (17), we argue that the probability of two SINRs of $\gamma_{(N), l}^{(1)}, \gamma_{(N), l}^{(2)}, \ldots, \gamma_{(N), l}^{\left(N_{s}\right)}$ being located in the same column is very small in the interfering case, especially when $N \gg N_{s}$. This is because the received signal power that acts as the desired signal for one user becomes the interfering signal for other users. For example, for a two-user case the received SINR of user 1 at relay node $j$ in the $l$ th hop is given by,

$$
\gamma_{2 j, l}=\frac{\omega_{1 j, l}}{\omega_{2 j, l}+\sigma^{2}}
$$

and the received SINR of user 2 at relay node $j$ in the same hop is given by

$$
\gamma_{2 j, l}=\frac{\omega_{2 j, l}}{\omega_{1 j, l}+\sigma^{2}}
$$

Note how the signal power that appears in the numerator in (21) changes to the interfering power that appears in the denominator in (22) and vice versa. This structure makes the probability of $\gamma_{1 j, l}$ and $\gamma_{2 j, l}$ being the two best SINRs in rows one and two, respectively, very small. As such, we can write a lower bound on the outage probability of user $u$ based on (9) as

$$
\begin{aligned}
& P_{o}^{(u)} \geq 1-\left(1-\mathbb{P}\left[\gamma_{u, L} \leq \gamma_{t h}\right]\right) \prod_{l=1}^{L-1}\left(1-\mathbb{P}\left[\gamma_{(N), l}^{(u)} \leq \gamma_{t h}\right]\right) \\
& \stackrel{(a)}{=} 1-\left(1-\mathbb{P}\left[\gamma_{u, L} \leq \gamma_{t h}\right]\right) \prod_{l=1}^{L-1}\left(1-\left(\mathbb{P}\left[\gamma_{u j, l} \leq \gamma_{t h}\right]\right)^{N}\right)
\end{aligned}
$$

where $\gamma_{u, L}$ denotes the SINR of user $u$ at the last hop and $j \in\{1,2, \ldots, N\}$. The result in (a) follows from the fact that $\gamma_{(N), l}^{(u)}$ is the maximum of $N$ i.i.d. SINRs. Based on the proof of $\mathbb{P}\left[\gamma_{u j, l} \leq \gamma_{t h}\right]$ given in Appendix $\mathrm{C}$, the outage probability of user $u$ can be lower bounded as (24), given at the top of next page, where $\Phi_{l}=P /\left(d_{u j, l}^{(u)}\right)^{\beta}$ and $\Theta_{l}=\frac{\gamma_{t h}^{q}\left(\frac{m}{\Phi_{l}}\right)^{\left(N_{s}-1\right) m}}{\Gamma(q+1) \Gamma\left(\left(N_{s}-1\right) m+q\right)}$. In section VI, we illustrate that the expression in (24) provides an accurate lower bound for the outage probability of user $u$ when the number of relays is large compared to the number of users.

\section{B. Outage Probability Saturation}

In the high SINR regime this outage probability lower bound saturates due to the effect of interference. This can be illustrated as follows. Let us reexpress the exponential function in (24) in a series expansion as

$$
\begin{aligned}
& P_{o}^{(u)} \geq 1-\left(\sum_{q=0}^{m-1} \sum_{k=0}^{q} \sum_{r=0}^{\infty} \Upsilon\left(\frac{\left(d_{u j, L}^{(u)}\right)^{\beta}}{P}\right)^{r+q-k}\right) \\
& \quad \times \prod_{l=1}^{L-1}\left(1-\left(1-\sum_{q=0}^{m-1} \sum_{k=0}^{q} \sum_{r=0}^{\infty} \Upsilon\left(\frac{\left(d_{u j, l}^{(u)}\right)^{\beta}}{P}\right)^{r+q-k}\right)^{N}\right),
\end{aligned}
$$

where $\Upsilon=\left(\begin{array}{l}q \\ k\end{array}\right) \frac{(-1)^{r} m^{r+q-k} \gamma_{t h}^{r+q} \sigma^{2(r+q-k)} \Gamma\left(\left(N_{s}-1\right) m+k\right)}{r !\left(\gamma_{t h}+1\right)^{\left(N_{s}-1\right) m+k} \Gamma(q+1) \Gamma\left(\left(N_{s}-1\right) m\right)}$ contains all the terms that are independent from $P$. Then we consider the lowest exponent for $1 / P$ which gives $r=0$ and $k=q$ as

$$
\begin{aligned}
P_{o}^{(u)} \geq 1- & \left(\sum_{q=0}^{m-1} \Upsilon_{0}+\mathcal{O}\left(P^{-1}\right)\right) \\
& \times\left(1-\left(1-\sum_{q=0}^{m-1} \Upsilon_{0}+\mathcal{O}\left(P^{-1}\right)\right)^{N}\right)^{L-1}
\end{aligned}
$$

where $\Upsilon_{0}=\sum_{q=0}^{m-1} \frac{\gamma_{t h}^{q} \Gamma\left(\left(N_{s}-1\right) m+q\right)}{\left(\gamma_{t h}+1\right)^{\left(N_{s}-1\right) m+q} \Gamma(q+1) \Gamma\left(\left(N_{s}-1\right) m\right)}$. Then, applying the binomial expansion to expand the powers followed by some mathematical manipulations the smallest order term of $P$ in (26) can be separated as

$$
P_{o}^{(u)} \geq 1-\Upsilon_{0}\left(1-\left(1-\Upsilon_{0}\right)^{N}\right)^{L-1}+\mathcal{O}\left(P^{-1}\right)
$$

As can be seen by (27) the smallest order of $P$ is zero and the outage probability lower bound has an error floor in the high SINR regime.

\section{NumericAl AND Simulation RESUlts}

In this section, we present simulation results to validate our analysis and evaluate the performance of the sub-optimal DRS scheme. All nodes have the same power $P$ which varies between $15 \mathrm{dBm}-30 \mathrm{dBm}(\approx 30 \mathrm{~mW}-10 \mathrm{~W})$, and the noise variance is set to one. We consider equal distances between the $L$ clusters, i.e., $d_{i j, l}=d / L$, where $d$ is the the distance between the source and the destination which is set to $2 \mathrm{~km}$. The value of the path loss is $140 \mathrm{~dB}$ for the first kilometer with path-loss exponent $\beta=3.8$ which corresponds to builtup areas. The SNR threshold $\gamma_{t h}$ is set to $5 \mathrm{~dB}$ unless otherwise stated.

Fig. 2 plots the simulated outage probability with transmit power for the DRS scheme with those of the optimal (exhaustive search) RS, naive RS and random RS schemes of a two-user network $\left(N_{s}=2\right)$ with $N=2$ and $L=3$ over Rayleigh fading $(m=1)$ channels. In the random RS, each user randomly chooses a relay at each hop without conflict. For the DRS, ORS, or random RS schemes, both users have the same outage probability. For the naive RS scheme, user 1 first selects the best path in each hop (similar to the DRS). Then, user 2 selects its best path that does not conflict with 


$$
\begin{aligned}
P_{o}^{(u)} & \geq 1-\left(\exp \left(-\frac{m \gamma_{t h}}{\Phi_{L}}\right) \sum_{q=0}^{m-1} \sum_{k=0}^{q}\left(\begin{array}{l}
q \\
k
\end{array}\right) \frac{\Theta_{L} \sigma^{2(q-k)} \Gamma\left(\left(N_{s}-1\right) m+k\right)}{\left(\frac{m \gamma_{t h}}{\Phi_{L}}+\frac{m}{\Phi_{L}}\right)^{\left(N_{s}-1\right) m+k}}\right) \\
& \times \prod_{l=1}^{L-1}\left(1-\left(1-\exp \left(-\frac{m \gamma_{t h} \sigma^{2}}{\Phi_{l}}\right) \sum_{q=0}^{m-1} \sum_{k=0}^{q}\left(\begin{array}{l}
q \\
k
\end{array}\right) \frac{\Theta_{l} \sigma^{2(q-k)} \Gamma\left(\left(N_{s}-1\right) m+k\right)}{\left(\frac{m \gamma_{t h}}{\Phi_{l}}+\frac{m}{\Phi_{l}}\right)^{\left(N_{s}-1\right) m+k}}\right)^{N}\right),
\end{aligned}
$$

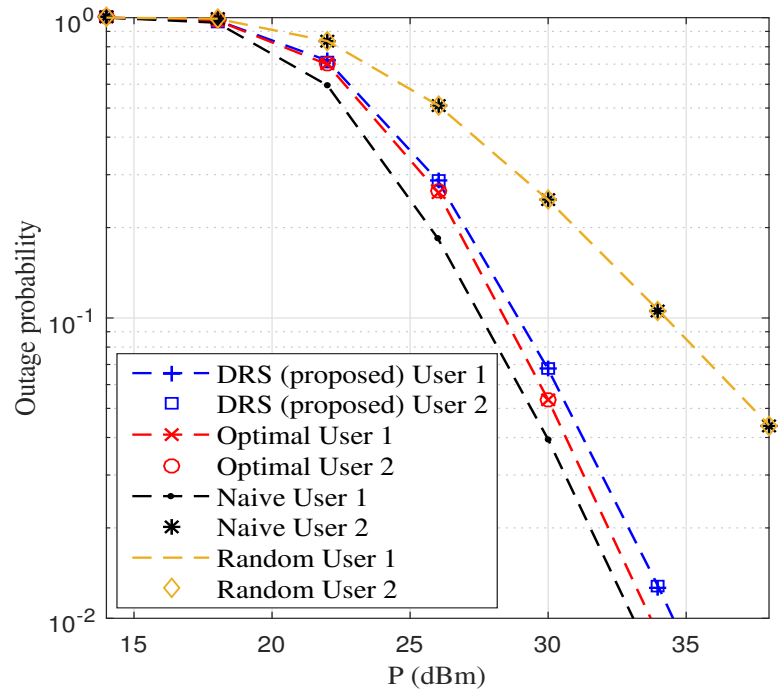

Fig. 2. User outage probability for two-user networks for ORS, DRS, naive $\mathrm{RS}$, and random RS schemes when $N_{s}=2, N=2$, and $L=3$.

user 1 . As such, the outage probabilities of user 1 and user 2 are different. user 1 actually achieves the performance of the single-user DRS since it has all $N$ relays to choose from. As such, in this example the performance of user 1 with naive RS is even larger than the ORS scheme. User 2 has a worse performance since it has only $(N-1)$ relays to choose from. This demonstrates the unfairness of the naive RS scheme. The DRS, ORS and naive RS (user 1) schemes achieve the same diversity order of two. The naive scheme (user 2) and the random RS provide the same performance and achieve a diversity order of only one because when there are only two relays, user 2 has no option for the RS as one relay has already been selected by user 1 . At $P=30 \mathrm{dBm}$, while the ORS (user 1 or user 2) and naive RS (user 1) outperform the DRS (user 1 or user 2) by approximately $1 \mathrm{~dB}$ and $2.5 \mathrm{~dB}$, respectively, the DRS (user 1 or user 2) outperforms naive RS (user 2) and random RS (user 1 or user 2) by approximately $8 \mathrm{~dB}$. Since both users have the same outage probability, the DRS scheme provides fairness among users.

Fig. 3 plots the outage probability with transmit power for each user of a two-user network with $N=2,3$ or 4 , and $L=4$ over Nakagami- $m$ fading channels when $m=2$. The simulation curves are shown as discrete marks and the exact analytical outage probability curves generated from (15) are

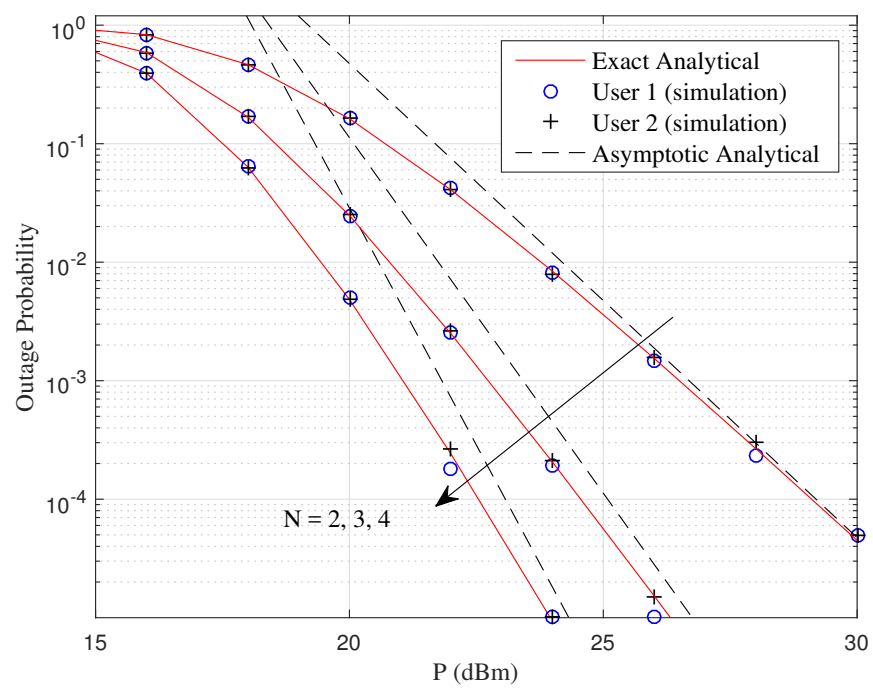

Fig. 3. User outage probability for two-user networks with $N=2,3,4$, $m=2$ and $L=4$ for the proposed DRS scheme.

shown as continuous lines. The asymptotic analytical outage probability lines generated from (32) are shown as dashed lines. For the entire simulated power range, we can see that our exact analytical results match the simulation for all network settings and the system archives a diversity order of 2, 3 and 4 as we change $N=2,3$ and 4 . This confirms the accuracy of our analysis for two-user multihop relay networks. This figure also shows the fairness of the DRS scheme as both users achieve similar outage probability performances.

Fig. 4 plots the outage probability with the transmit power for user 1 of a three-user network $\left(N_{s}=3\right)$ with $N=3$ or 6 , and $L=3$ or 4 over Rayleigh fading $(m=1)$ channels. Only the simulated outage probability of user 1 is shown in the figure as all users have the same outage probability. The outage probability approximation in (32) is accurate for large $P$ which confirms the validity of our analysis of the diversity order and array gain. We also observe that the DRS scheme has diversity order 3 or 6 for $N=3$ or 6 , respectively, which is the full diversity order. We further investigate the effect of the number of hops on array gain. For $N=3$ at $P=$ $28 \mathrm{dBm}$, the $L=3$ has $13 \mathrm{~dB}$ loss in array gain compared with $L=4$. Similarly, for $N=6$ at $P=26 \mathrm{dBm}$, the $L=$ 3 has $27 \mathrm{~dB}$ loss in array gain compared with $L=4$, which is a significant performance loss. For this example, when the 


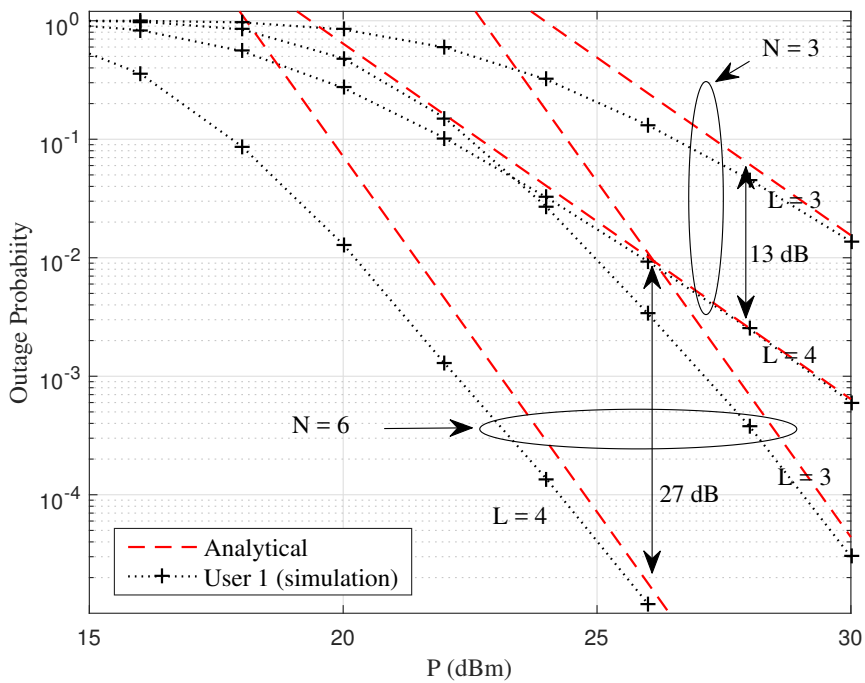

Fig. 4. User outage probability for multi-user networks with $N_{s}=3$, $N=3,6$, and $L=3,4$ for the proposed DRS scheme.

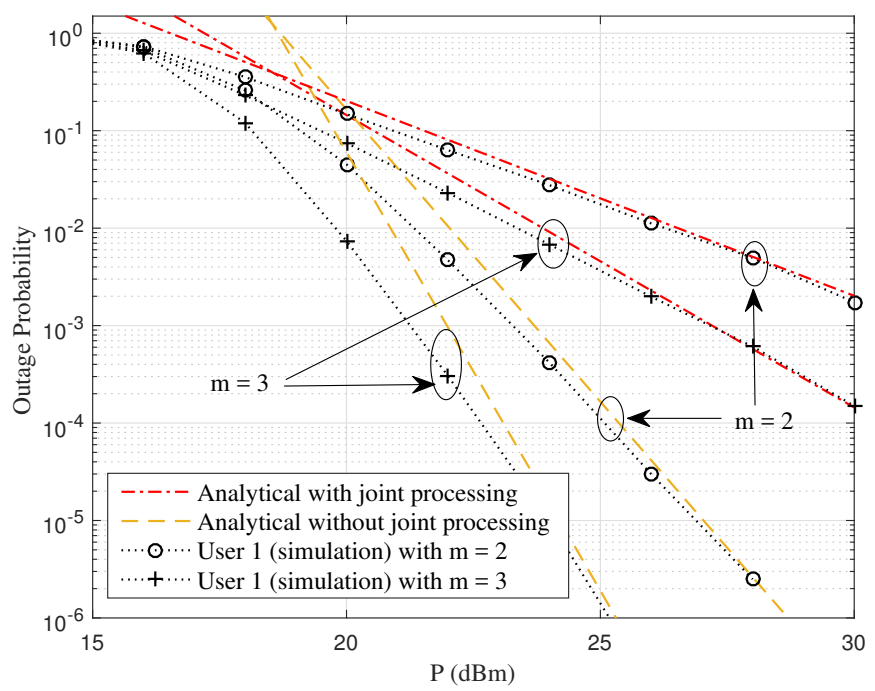

Fig. 5. User outage probability for multi-user networks with $N_{s}=3$, $N=3, m=2,3$ and $L=4$ for the proposed DRS scheme.

number of relays is doubled $(N=3$ to $N=6)$, the array gain is also approximately doubled. Furthermore, the distance dependent path-loss has a major impact on the user outage as well.

Fig. 5 plots the outage probability with the transmit power for user 1 of a three-user network $\left(N_{s}=3\right)$ for $N=3$ and $L=4$ over Nakagami- $m$ fading channels when $m=2$ or 3 . Based on the RS at last two hops, we consider two scenarios: i) with joint processing, and ii) without joint processing. With joint processing, the DRS has diversity order 6 and 9 when $m=2$ and 3, respectively, which is the full diversity order $N m$, confirming the accuracy of (32). Without joint processing, the DRS has diversity order 2 and 3 when $m=2$ and 3, respectively, which is a reduced diversity order $m$ which

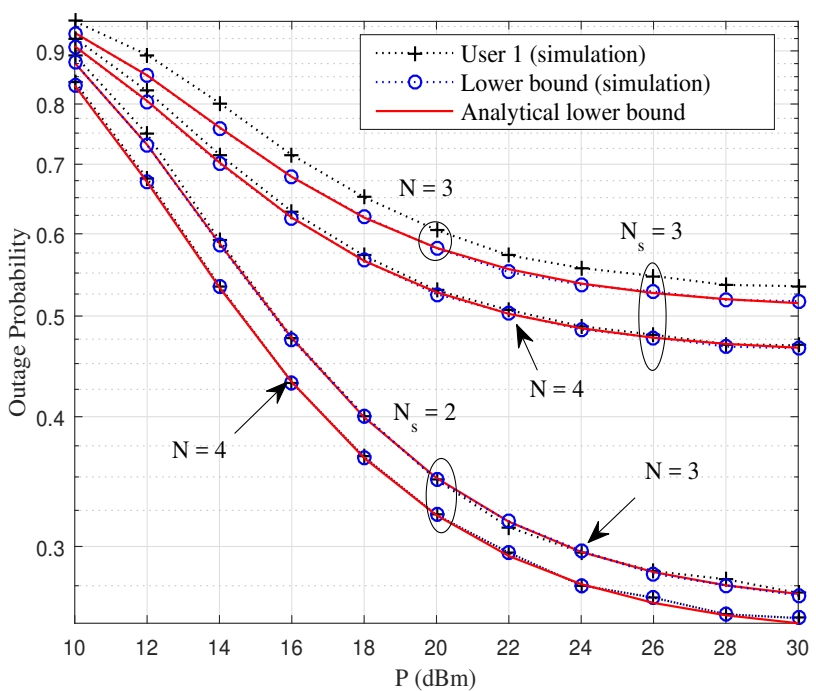

Fig. 6. User outage probability lower bound for interfering multi-user networks with $N_{s}=2,3, N=3,4, m=1$ and $L=4$ for the proposed DRS scheme.

is independent of number of relays $N$. This also confirms the accuracy of (12), and clearly illustrates the importance of joint processing in DRS scheme. For example, when we apply joint processing at $m=2$ we can achieve $P_{o}^{(u)}=2 \times 10^{-3}$, with approximately $7 \mathrm{dBm}$ less power when compared to without joint processing. Similarly, when we apply joint processing at $m=3$ we can achieve $P_{o}^{(u)}=2 \times 10^{-3}$, with approximately $5 \mathrm{dBm}$ less power when compared to without joint processing.

Fig. 6 plots the outage probability lower bound for a multiuser multihop relay network with interfering users, for $N_{s}=2$ and $3, N=3$ and 4 and $L=4$. Assuming a Rayleigh faded environment the Nakagami shape parameter $m$ is set to one. The SINR threshold $\gamma_{t h}$ is set to $-5 \mathrm{~dB}^{2}$. The simulation curves for the exact outage probability and the lower bound are shown as dotted lines with discrete marks and the analytical outage probability lower bound curves generated from (24) are shown as continuous lines. For the entire simulated power range, we can see that our analytical lower bound exactly matches the simulation lower bound for all network settings. This confirms the accuracy of our analysis. For $N_{s}=2$, we observe that the lower bound is almost on top of the exact outage probability curve when $N=3$ and 4 . For $N_{s}=3$, we observe that the lower bound is lose when $N=3$ but it is almost on top of the exact outage probability as soon $N$ is as large as 4 . We also observe that due to the effect of interference the outage probability performance is significantly degraded. In the high SINR regime the outage enters a saturation highlighting the importance in interference cancellation in multi-user relay networks.

\footnotetext{
${ }^{2}$ Note that the outage probability is significantly degraded due to the effect of interference. As such, we selected a lower SINR threshold to clearly illustrate the effect of interference on outage probability.
} 


\section{CONCLUSION}

The relay selection problem in a network with multiple source-destination pairs with multiple hops is analysed in this paper. A sub-optimal DRS scheme is proposed and analysed under general channel conditions with Nakagami- $m$ fading and path loss. As opposed to ORS whose complexity increases exponentially with the number of hops, the complexity of the proposed decentralized RS scheme is quadratic in the number of users, linear in the number of relays and independent of the number of hops. Based on a rigorous theoretical analysis the proposed scheme was shown to achieve full spatial diversity. For a two-user network the exact closed-form expression of the outage probability was derived and tested against simulations. Extending the analysis to interfering relay networks, an accurate lower bound on the outage probability was derived in closed-form. Due to the effect of interference the outage probability was shown to enter a saturation in the high SINR regime.

An interesting extension to this work is to apply this system model to a cognitive radio network and study routing protocols for path selection. While in [23]-[25] the authors consider multihop relaying in cognitive radio networks only a single secondary source and destination is considered. However, extending to multiple secondary sources and secondary destinations would formulate interesting research problems. The analysis in this paper was based on DF relaying. An extension to AF relaying would formulate another challenging analytical problem. While error accumulation in AF relaying limits its extension to multihop relaying, a comparison would lead to interesting insights. It is also desirable to extend the analysis to full-duplex relays to gain more interesting insights.

\section{APPENDIX A}

\section{PROOF OF THEOREM 1}

In this section we provide a detailed proof of Theorem 1 . Note that the SNR elements of $\boldsymbol{\Gamma}_{l}$ are i.i.d.. Thus there are in total $\left(N_{s} N\right)$ ! possible orderings of the SNR elements, each with probability $1 /\left(N_{s} N\right)$ !. For any given $k$, we can count the number of orderings that result in $\gamma_{u, l}=\gamma_{(k), l}$ and denote it as $\varepsilon_{k}$. Thus $\mathbb{P}\left[\gamma_{u, l}=\gamma_{(k), l}\right]=\varepsilon_{k} /\left(N_{s} N\right)$ !. For the DRS scheme, only the relay ordering matters i.e., $\varepsilon_{k}$ depends only on $N_{s}, N$ (the dimensions of $\boldsymbol{\Gamma}_{l}$ ) and $k$, and is independent of $F_{\hat{\gamma}_{i j, l}}(x)$ which is the CDF of each element of $\boldsymbol{\Gamma}_{l}$. Therefore, $\mathbb{P}\left[\gamma_{u, l}=\right.$ $\left.\gamma_{(k), l}\right]$ is independent of $F_{\dot{\gamma}_{i j, l}}(x)$, and does not include $P$.

Based on the previous outage probability analysis in (10), we can write

$$
\begin{aligned}
& P_{o}^{(u)}=1 \\
& -\prod_{l=1}^{L-1}\left(1-\sum_{k=1}^{N_{s}} \sum_{i=0}^{N-N+1} \Psi_{l k i}\left(F_{\dot{\gamma}_{i j, l}}\left(\gamma_{t h}\right)\right)^{N_{S} N-k+i+1}\right),
\end{aligned}
$$

where (28) follows by substituting $F_{\gamma_{(k), l}}\left(\gamma_{t h}\right)$ in (7) into (10) and $\Psi_{l k i}=\frac{\mathbb{P}\left[\gamma_{u, l}=\gamma_{(k), l}\right]\left(N_{s} N\right) !\left(\begin{array}{c}k-1 \\ i\end{array}\right)}{(-1)^{i}\left(N_{s} N-k+i+1\right)(k-1) !\left(N_{s} N-k\right) !}$. From (28) we learn that only the term $F_{\dot{\gamma}_{i j, l}}\left(\gamma_{t h}\right)$ depends on $P$. Therefore, we proceed to reexpress $F_{\dot{\gamma}_{i j, l}}\left(\gamma_{t h}\right)$ based on
(6) and (4) to write (29) given at the top of the next page, where we have separated the product terms in (28) based on the outage probability of the first $L-2$ hops and the last two hops. Next, we use the infinite series expansion of the incomplete gamma function, which is given by $\gamma\left(m, \frac{m x}{\tilde{\Omega}_{l}}\right)=\left(\frac{m x}{\tilde{\Omega}_{l}}\right)^{m} \exp \left(\frac{-m x}{\tilde{\Omega}_{l}}\right) \sum_{q=0}^{\infty} \frac{\left(m x / \tilde{\Omega}_{l}\right)^{q}}{m(m+1) \ldots(m+q)}$, and express the exponential function therein using [28, eq. (1.211)] to write (30) given at the top of next page. Based on (30), we identify that the effective terms at high power region, i.e., the terms with the lowest exponent for $1 / P$ as $P \rightarrow \infty$, are obtained when $t=q=i=0$ and $k=N_{s} N-N+1$. Using this, and the fact that $\tilde{\Omega}_{l}=\Omega=P /\left((d / L)^{\beta} \sigma^{2}\right)$ for $l=1, \cdots,(L)$, we next apply the binomial expansion and write the product of $L-2$ terms as a summation given by (31) at the top of the next page. Note that in (31) we have only expressed the dominant terms in the high SNR regime. As $\mathbb{P}\left[\gamma_{u, l}=\gamma_{\left(N_{s} N-N+1\right), l}\right]=\mathbb{P}\left[\gamma_{u, z}=\gamma_{\left(N_{s} N-N+1\right), z}\right]$ for any $l \neq z, l \in 1,2, \ldots, L-1$ we can further simplify (31) as

$$
\begin{aligned}
& P_{o}^{(u)} \\
& \underbrace{\frac{\left(L-2+2^{N}\right) \mathbb{P}\left[\gamma_{u, l}=\gamma_{\left(N_{s} N-N+1\right), l}\right]\left(N_{s} N\right) ! m^{m N}}{N !\left(N_{s} N-N\right) !(m !)^{N}\left(\gamma_{t h}\left(\frac{d}{L}\right)^{\beta} \sigma^{2}\right)^{-m N}}}_{\epsilon} P^{-m N} \\
& +\mathcal{O}\left(\frac{1}{P^{m+1}}\right)
\end{aligned}
$$

where

$\mathbb{P}\left[\gamma_{u, l}=\gamma_{\left(N_{s} N-N+1\right), l}\right]=\left\{\begin{array}{cl}\frac{1}{N_{s}} \prod_{i=1}^{N-1} \frac{N-i}{N_{s} N-i} ; & N_{s}<N \\ \frac{2}{N_{s}} \prod_{i=1}^{N-1} \frac{N-i}{N_{s} N-i} ; & N_{s}=N,\end{array}\right.$

for all $l \in 1,2, \ldots, L-1$ and the array gain of user $u$ denoted as $\epsilon$.

\section{APPENDIX B \\ EXAMPLE: DERIVATION OF $F_{\gamma_{(k), l}}(x)$ IN INTERFERING RELAY NETWORKS}

In this section, we provide a detailed derivation of $F_{\gamma_{(k), l}}(x)$ for a simple example. Let us consider the $l$ th hop of a relay network with two users and two relays in each hop. Let the channel be subjected to Rayleigh fading. Assuming that the users interfere with each other we can write the received SINR matrix as

$$
\boldsymbol{\Gamma}_{l}=\left[\begin{array}{c}
\gamma_{11, l} \gamma_{12, l} \\
\gamma_{21, l} \gamma_{22, l}
\end{array}\right]
$$

where $\gamma_{u j, l}=\frac{\omega_{u j, l}}{\omega_{v j, l}+\sigma^{2}}, v \in\{1,2\}, v \neq u$. Based on (20) the CDF of the largest, second largest and the third largest SINRs from among the four SINRs in (34) can be written as follows. For the ease of notation let $\mathcal{S}=\left\{\gamma_{11, l}, \gamma_{21, l}, \gamma_{12, l}, \gamma_{22, l}\right\}$.

$$
F_{\gamma_{(1), l}}(x)=\sum_{\gamma_{p}, \gamma_{q}, \gamma_{r}, \gamma_{s} \in \mathcal{S}} F_{\gamma_{p}, \gamma_{q}, \gamma_{r}, \gamma_{s}}(x, x, x, x)
$$




$$
\begin{aligned}
& P_{o}^{(u)}=1-\left[\prod_{l=1}^{L-2}\left(1-\sum_{k=1}^{N_{s} N-N+1} \sum_{i=0}^{k-1} \Psi_{l k i}\left(\frac{\gamma\left(m, \frac{m \gamma_{t h}}{\tilde{\Omega}_{l}}\right)}{\Gamma(m)}\right)^{N_{S} N-k+i+1}\right)\right. \\
& \left.\times\left(1-\sum_{k=1}^{N_{s}} \sum_{i=0}^{N-N+1} \Psi_{(L-1) k i}\left(1-\left(1-\frac{\gamma\left(m, \frac{m \gamma_{t h}}{\tilde{\Omega}_{L-1}}\right)}{\Gamma(m)}\right)\left(1-\frac{\gamma\left(m, \frac{m \gamma_{t h}}{\tilde{\Omega}_{L}}\right)}{\Gamma(m)}\right)\right)^{N_{S} N-k+i+1}\right)\right]
\end{aligned}
$$

$$
\begin{aligned}
& P_{o}^{(u)}=1-\left[\prod _ { l = 1 } ^ { L - 2 } \left(1-\sum_{k=1}^{N_{s} N-N+1} \sum_{i=0}^{k-1} \Psi_{l k i}\left(\frac{\left.\left(\frac{m \gamma_{t h}}{\Omega_{l}}\right)^{m} \sum_{t=0}^{\infty} \sum_{q=0}^{\infty} \frac{(-1)^{t}\left(\frac{m \gamma_{t h}}{\Omega_{l}}\right)^{t+q}}{\Gamma(m)}\right)^{\left.N_{S} N-k+1\right) \ldots(m+q)}}{\Gamma(m)}\right)\right.\right. \\
& \times\left(1-\sum_{k=1}^{N_{s}} \sum_{i=0}^{N-N+1} \Psi_{(L-1) k i}\left(1-\left(1-\frac{\left.\left(\frac{m \gamma_{t h}}{\tilde{\Omega}_{L-1}}\right)^{m} \sum_{t=0}^{\infty} \sum_{q=0}^{\infty} \frac{(-1)^{t}\left(\frac{m \gamma_{t h}}{\bar{\Omega}_{L-1}}\right)^{t+q}}{\overline{t ! m(m+1) \ldots(m+q)}}\right)}{\Gamma(m)}\right)\right.\right.
\end{aligned}
$$



$$
\begin{aligned}
P_{o}^{(u)} \approx \sum_{l=1}^{L-2} \frac{\mathbb{P}\left[\gamma_{u, l}=\gamma_{\left(N_{s} N-N+1\right), l}\right]\left(N_{s} N\right) ! m^{m N}}{N !\left(N_{s} N-N\right) !(m !)^{N}}\left(\frac{\gamma_{t h}\left(\frac{d}{L}\right)^{\beta} \sigma^{2}}{P}\right)^{m N} & \\
& \quad+\frac{2^{N} \mathbb{P}\left[\gamma_{u, L-1}=\gamma_{\left(N_{s} N-N+1\right), L-1}\right]\left(N_{s} N\right) ! m^{m N}}{N !\left(N_{s} N-N\right) !(m !)^{N}}\left(\frac{\gamma_{t h}\left(\frac{d}{L}\right)^{\beta} \sigma^{2}}{P}\right)^{m N}+\mathcal{O}\left(\frac{1}{P^{m N+1}}\right) .
\end{aligned}
$$

$$
\begin{aligned}
& F_{\gamma_{(2), l}}(x)=\sum_{\gamma_{p}, \gamma_{q}, \gamma_{r} \in \mathcal{S}} F_{\gamma_{p}, \gamma_{q}, \gamma_{r}}(x, x, x) \\
& -3 \sum_{\gamma_{p}, \gamma_{q}, \gamma_{r}, \gamma_{s} \in \mathcal{S}} F_{\gamma_{p}, \gamma_{q}, \gamma_{r}, \gamma_{s}}(x, x, x, x), \\
& F_{\gamma_{(3), l}}(x) \\
& =\sum_{\gamma_{p}, \gamma_{q} \in \mathcal{S}} F_{\gamma_{p}, \gamma_{q}}(x, x)-2 \sum_{\gamma_{p}, \gamma_{q}, \gamma_{r} \in \mathcal{S}} F_{\gamma_{p}, \gamma_{q}, \gamma_{r}}(x, x, x) \\
& +3 \sum_{\gamma_{p}, \gamma_{q}, \gamma_{r}, \gamma_{s} \in \mathcal{S}} F_{\gamma_{p}, \gamma_{q}, \gamma_{r}, \gamma_{s}}(x, x, x, x) .
\end{aligned}
$$

As such, we need the joint CDF of all possible combinations of two, three and four SINRs in (34) in order to find the above order statistics. The joint CDF of two independent SINRs in $\mathcal{S}$, which we denote by $F_{(\text {ind })}(x, x)$, can be evaluated following the steps in Appendix $\mathrm{C}$ which results in

$$
F_{\text {(ind) }}(x, x)=\left(1-\frac{\exp \left(-x \sigma^{2}\right)}{1+x}\right)^{2} .
$$

The joint CDF of two correlated SINRs in $\mathcal{S}$, which we denote by $F_{\text {(corr) }}(x, x)$, can also be found based on some mathematical manipulations as given in (39). Then the expressions for
$F_{\gamma_{(1), l}}(x), F_{\gamma_{(2), l}}(x)$ and $F_{\gamma_{(3), l}}(x)$ can be expressed in closed form in-terms of $F_{\text {(ind) }}(x, x)$ and $F_{\text {(corr) }}(x, x)$ as

$$
\begin{aligned}
& F_{\gamma_{(1), l}}(x)=\left(F_{(\text {corr })}(x, x)\right)^{2} \\
& F_{\gamma_{(2), l}}(x)=4\left(F_{(\text {ind })}(x, x)\right)\left(F_{(\text {corr })}(x, x)\right)^{2} \\
& -3\left(F_{\text {(corr) }}(x, x)\right)^{2} \text {, } \\
& F_{\gamma_{(3), l}}(x)=4\left(F_{(\text {ind })}(x, x)\right)+2\left(F_{(\text {corr })}(x, x)\right) \\
& -2\left(F_{\text {(ind })}(x, x)\right)\left(F_{(\text {corr })}(x, x)\right)^{2}+3\left(F_{(\text {corr })}(x, x)\right)^{2} \text {. }
\end{aligned}
$$

Note that based on (20), (38) and (39) the ordered SINRs $F_{\gamma_{(1), l}}(x), F_{\gamma_{(2), l}}(x) \ldots F_{\gamma_{(N+1), l}}(x)$ required for a two-user network with $N$ relays can be found. However, when the number of users is large deriving general expressions for order statistics is mathematically challenging as we have to consider a large number of subsets of joint CDFs.

\section{APPENDIX C}

\section{PROOF OF $\mathbb{P}\left[\gamma_{u j, 1} \leq \gamma_{t h}\right]$}

In this section we derive an expression for $\mathbb{P}\left[\gamma_{u j, 1} \leq \gamma_{t h}\right]$. First, we reexpress $\gamma_{u j, 1}$ based on (17) and rewrite $\mathbb{P}\left[\gamma_{u j, 1} \leq\right.$ 


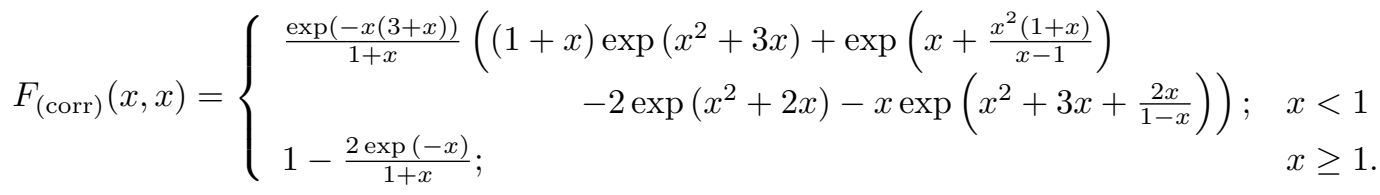

$\left.\gamma_{t h}\right]$ as

$$
\begin{aligned}
& \mathbb{P}\left[\gamma_{u j, 1} \leq \gamma_{t h}\right]=\mathbb{P}\left[\omega_{u j, l} \leq \gamma_{t h}\left(\sum_{k=1, k \neq u}^{N_{s}} \omega_{k j, l}+\sigma^{2}\right)\right] \\
& =E_{\Delta}\left[1-\exp \left(-\frac{m \gamma_{t h}\left(\Delta+\sigma^{2}\right)}{\Phi_{l}}\right) \sum_{q=0}^{m-1} \frac{\left(\frac{m \gamma_{t h}\left(\Delta+\sigma^{2}\right)}{\Phi_{l}}\right)^{q}}{\Gamma(q+1)}\right],
\end{aligned}
$$

where $E_{\Delta}[$.$] indicates the expectation taken over the distribu-$ tion of $\Delta=\sum_{k=1, k \neq u}^{N_{s}} \omega_{k j, l}$. Note that $\omega_{k j, l}$ has a gamma distribution with shape parameter $m$ and rate parameter $\left(m / \Phi_{l}\right)$. The sum of $\left(N_{s}-1\right)$ random variables with such independent and identical gamma distributions again results in a new gamma distribution with shape parameter $\left(N_{s}-1\right) m$ and rate parameter $\left(m / \Phi_{l}\right)$. As such, the expectation in (43) can be reexpressed as

$$
\begin{aligned}
& \mathbb{P}\left[\gamma_{u j, 1} \leq \gamma_{t h}\right]=1-\exp \left(-\frac{m \gamma_{t h} \sigma^{2}}{\Phi_{l}}\right) \sum_{q=0}^{m-1}\left[\Theta_{l}\right. \\
& \left.\times \int_{0}^{\infty}\left(\Delta+\sigma^{2}\right)^{q} \Delta^{\left(N_{s}-1\right) m-1} \exp \left(-\frac{\Delta m}{\Phi_{l}}\left(1+\gamma_{t h}\right)\right) d \Delta\right] .
\end{aligned}
$$

Expanding $\left(\Delta+\sigma^{2}\right)^{q}$ using binomial expansion and solving the resultant integral based on [28, eq. 3.351,3] results in the closed-form expression fo $\mathbb{P}\left[\gamma_{u j, 1} \leq \gamma_{t h}\right]$ given by

$$
\begin{aligned}
& \mathbb{P}\left[\gamma_{u j, 1} \leq \gamma_{t h}\right]=1-\left[\exp \left(-\frac{m \gamma_{t h} \sigma^{2}}{\Phi_{l}}\right)\right. \\
& \left.\times \sum_{q=0}^{m-1} \sum_{k=0}^{q}\left(\begin{array}{l}
q \\
k
\end{array}\right) \frac{\Theta_{l} \sigma^{2(q-k)} \Gamma\left(\left(N_{s}-1\right) m+k\right)}{\left(\frac{m \gamma_{t h}}{\Phi_{l}}+\frac{m}{\Phi_{l}}\right)^{\left(N_{s}-1\right) m+k}}\right] .
\end{aligned}
$$

\section{REFERENCES}

[1] F. Gomez-Cuba, R. Asorey-Cacheda, and F. J. Gonzalez-Castano, A survey on cooperative diversity for wireless networks, IEEE Commun. Surveys Tuts., vol. 14, no. 3, pp. 822-835, Third 2012.

[2] D. Gesbert, S. Hanly, H. Huang, S. Shamai, O. Simeone, and W. Yu, Multi-cell MIMO cooperative networks: A new look at interference, IEEE J. Sel. Areas Commun., vol. 28, pp. 1380-1408, Dec. 2010.

[3] P. Wang, H. Wang, L. Ping, and X. Lin, On the capacity of MIMO cellular systems with base station cooperation, IEEE Trans. Wireless Commun., vol. 10, p. 3720-3731, Nov. 2011.

[4] O. Simeone, N. Levy, A. Sanderovich, O. Somekh, B. M. Zaidel, H. V. Poor, and S. Shamai, Cooperative wireless cellular systems: An information-theoretic view, Foundations and Trends in Communications and Information Theory, vol. 8, pp. 1-177, Jan. 2011.

[5] J. Laneman, D. Tse, and G. Wornell, Cooperative diversity in wireless networks: Efficient protocols and outage behavior, IEEE Trans. Inf. Theory, vol. 50, pp. 3062-3080, Dec. 2004.

[6] A. Sendonaris, E. Erkip, and B. Aazhang, User cooperation diversity. Part I: System description, IEEE Trans. Commun., vol. 51, no. 11, pp. 1927-1938, Nov. 2003.
[7] X. Ge, S. Tu, G. Mao, C. X. Wang, and T. Han, 5G ultra-dense cellular networks, IEEE Wireless Communications, vol. 23, pp. 72-79, Feb. 2016.

[8] E. Koyuncu, Y. Jing, and H. Jafarkhani, Beamforming in wireless relay networks with quantized feedback, IEEE J. Sel. Areas Commun., vol. 26, pp. 1429-1439, Oct. 2008.

[9] A. Bletsas, A. Khisti, D. P. Reed, and A. Lippman, A simple cooperative diversity method based on network path selection, IEEE J. Sel. Areas Commun., vol. 24, pp. 659-672, Mar. 2006.

[10] V. Sreng, H. Yanikomeroglu, and D. D. Falconer, Relay selection strategies in cellular networks with peer-to-peer relaying, in IEEE Veh. Technol. Conf., VTC 2003, Orlando, FL, Oct. 2003.

[11] D. Michalopoulos and G. Karagiannidis, Performance analysis of single relay selection in Rayleigh fading, IEEE Trans. Wireless Commun., vol. 7, no. 10, pp. 37183724, Oct. 2008.

[12] S. Atapattu and N. Rajatheva, PEP bound for multiple relay transmission over Nakagami-m fading channels, in Second Intl. Conf. on Indus. and Info. Sys. (ICIIS 2007), Sri Lanka, Aug. 2007.

[13] A. S. Ibrahim, A. Sadek, W. Su, and K. J. R. Liu, Cooperative communications with relay-selection: When to cooperate and whom to cooperate with, IEEE Trans. Commun., vol. 7, pp. 2814-2827, Jul. 2008.

[14] Y. Jing and H. Jafarkhani, Single and multiple relay selection schemes and their achievable diversity orders, IEEE Trans. Wireless Commun., vol. 8, no. 3, pp. 1414-1423, Mar. 2009.

[15] S. Sharma, Y. Shi, Y. T. Hou, and S. Kompella, An optimal algorithm for relay node assignment in cooperative ad hoc networks, IEEE/ACM Trans. Netw., vol. 19, pp. 879-892, Jun. 2011.

[16] Q. Li, M. Yu, A. Pandharipande, X. Ge, J. Zhang, and J. Zhang, Performance of virtual full-duplex relaying on cooperative multi-path relay channels, IEEE Trans. Wireless Commun., vol. 15, pp. 3628-3642, May 2016.

[17] Q. Li, M. Yu, A. Pandharipande, and X. Ge, Outage analysis of cooperative two-path relay channels, IEEE Trans. Wireless Commun., vol. 15, pp. 3573-3578, May 2016.

[18] S. Atapattu, Y. Jing, H. Jiang, and C. Tellambura, Relay selection and performance analysis in multiple-user networks, IEEE J. Sel. Areas Commun., vol. 31, pp. 1517-1529, Aug. 2013.

[19] L. Zhang and L. J. Cimini, Hop-by-hop routing strategy for multihop decode-and-forward cooperative networks, in IEEE Wireless Communications and Networking Conference, WCNC 2008, Las Vegas, USA, May 2008, pp. 576-581.

[20] B. Gui, L. Dai, and L. J. Cimini, Routing strategies in multihop cooperative networks, IEEE Trans. Wireless Commun., vol. 8, pp. 843855, Feb. 2009.

[21] M. R. Bhatnagar, Performance analysis of a path selection scheme in multihop decode-and-forward protocol, IEEE Commun. Lett., vol. 16, pp. 1980-1983, Dec. 2012.

[22] M. R. Bhatnagar, R. K. Mallik, and O. Tirkkonen, Performance evaluation of best-path selection in a multihop decode-and-forward cooperative system, IEEE Trans. Veh. Technol., vol. 65, pp. 2722-2728, Apr. 2016.

[23] H. K. Boddapati, M. R. Bhatnagar, and S. Prakriya, Ad-hoc relay selection protocols for multi-hop underlay cognitive radio networks, in IEEE Globecom Workshops (Globecom 2016), Washington DC, USA, pp. 1-6, Dec. 2016.

[24] H. K. Boddapati, S. Prakriya, and M. R. Bhatnagar, Throughput analysis of cooperative multi-hop underlay CRNs with incremental relaying, in IEEE International Conference on Communications Workshops (ICC 2017), Paris, France, pp. 379-385, May 2017.

[25] H. K. Boddapati, M. R. Bhatnagar, and S. Prakriya, Performance analysis of cluster-based multi-hop underlay CRNs using max-link selection protocol, IEEE Transactions on Cognitive Communications and Networking, vol. IEEE Early Access Articles.

[26] C. Wang and M. Skoglund, Multi-user multi-hop relay networks: Transmission schemes and degrees of freedom, IEEE Trans. Wireless Commun., vol. 14, no. 8, pp. 4582-4596, Aug 2015.

[27] R. Senanayake, S. Atapattu, P. L. Yeoh, and J. Evans, Decentralized relay selection in two-user multihop decode-and-forward relay networks, 
in IEEE International Conference in Communications, ICC 2017, Paris, France, May 2017.

[28] I. Grashteyn and I. Ryzhik, Table of Integrals, Series, and Products, 7th ed. Academic Press, 2007.

[29] Z. Wang and G. B. Giannakis, A simple and general parameterization quantifying performance in fading channels, IEEE Trans. Commun., vol. 51, pp. 1389-1398, Aug. 2003.

[30] H. A. David and H. N. Nagaraja, Order Statistics, 3rd ed. John Wiley $\&$ sons, inc, publication, 2003.

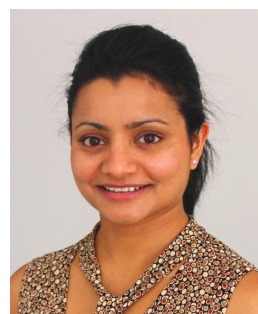

Rajitha Senanayake (S'11-M'16) received the B. E. degree in Electrical and Electronics Engineering from the University of Peradeniya, Sri Lanka, and the BIT degree in Information Technology from the University of Colombo, Sri Lanka, in 2009 and 2010, respectively. From 2009 to 2011, she was with the research and development team at Excel Technology, Sri Lanka. She received the Ph.D. degree in Electrical and Electronics Engineering from the University of Melbourne, Australia, in 2015. From 2015 to 2016 she was with the Department of Electrical and Computer Systems Engineering, Monash University, Australia. Currently she is a research fellow at the Department of Electrical and Electronics Engineering at the University of Melbourne, Australia. She is a recipient of the Australian Research Council Discovery Early Career Researcher Award. Her research interests are in cooperative communications, distributed antenna systems and Fog computing.

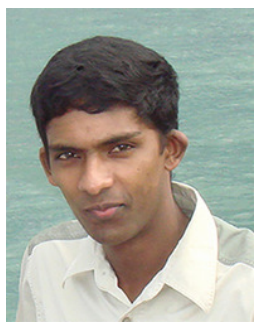

Saman Atapattu (M'14) received the B.Sc. degree in electrical and electronics engineering from the University of Peradeniya, Sri Lanka in 2003, the M.Eng. degree in telecommunications from Asian Institute of Technology, Thailand in 2007, and the $\mathrm{Ph} . \mathrm{D}$. degree in electrical engineering from the University of Alberta, Canada in 2013. He is currently a Research Fellow with the Department of Electrical and Electronic Engineering, the University of Melbourne, Australia. His general research interests include wireless communications and signal process-

ing.

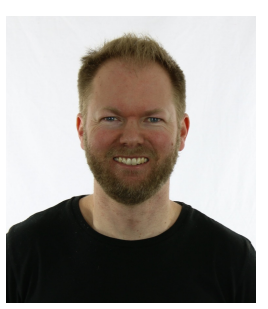

Jamie S. Evans (S'93-M'98) was born in Newcastle, Australia, in 1970. He received the B.S. degree in physics and the B.E. degree in computer engineering from the University of Newcastle, in 1992 and 1993, respectively, where he received the University Medal upon graduation. He received the M.S. and the Ph.D. degrees from the University of Melbourne, Australia, in 1996 and 1998, respectively, both in electrical engineering, and was awarded the Chancellor's Prize for excellence for his Ph.D. thesis. From March 1998 to June 1999, he was a Visiting Researcher in the Department of Electrical Engineering and Computer Science, University of California, Berkeley. Since returning to Australia in July 1999 he has held academic positions at the University of Sydney, the University of Melbourne and Monash University. He is currently a Professor and Deputy Dean in the Melbourne School of Engineering at the University of Melbourne. His research interests are in communications theory, information theory, and statistical signal processing with a focus on wireless communications networks.



Peter J. Smith (M'93-SM'01-F'15) received the B.Sc. degree in mathematics and the Ph.D. degree in statistics from the University of London, London, U.K., in 1983 and 1988, respectively. From 1983 to 1986, he was with the Telecommunications Laboratories, General Electric Company Hirst Research Centre. From 1988 to 2001, he was a Lecturer in statistics with the Victoria University of Wellington, Wellington, New Zealand. During 20012015, he was with the Department of Electrical and Computer Engineering, University of Canterbury, Christchurch, New Zealand. In 2015, he joined Victoria University of Wellington as Professor of statistics. His research interests include the statistical aspects of design, modeling, and analysis for communication systems, cognitive radio, massive multiple-inputmultiple-output, and millimeter-wave systems. 